\title{
Homogenization of the Navier-Stokes Equations with a Slip Boundary Condition
}

\author{
GRÉGOIRE ALLAIRE \\ Courant Institute \\ and \\ Commissariat à l'Energie Atomique, Saclay
}

\begin{abstract}
This paper deals with the homogenization of the Stokes or Navier-Stokes equations in a domain containing periodically distributed obstacles, with a slip boundary condition (i.e., the normal component of the velocity is equal to zero, while the tangential velocity is proportional to the tangential component of the normal stress). We generalize our previous results (see [1]) established in the case of a Dirichlet boundary condition; in particular, for a so-called critical size of the obstacles (equal to $\varepsilon^{3}$ in the threedimensional case, $\varepsilon$ being the inter-hole distance), we prove the convergence of the homogenization process to a Brinkman-type law.
\end{abstract}

\section{Introduction}

In a recent article (see [1]) we addressed the problem of the homogenization of the Stokes or Navier-Stokes equations, with a Dirichlet boundary condition, in open sets perforated with tiny holes. The present paper is devoted to the generalization of that previous study to the case of a slip boundary condition (the normal component of the velocity is equal to zero, but the tangential component is proportional to the tangential component of the normal stress). Roughly speaking, all the results of [1] still hold true, including the construction of an extension of the pressure, and the three different limit regimes (Darcy, Brinkman, Stokes). In both cases the critical size of the holes (leading to the homogenized Brinkman-type law) is the same, but the matrix $M$ which appears in the limit is different. From a mathematical view point this paper shows that the same type of results are obtained either if all the $N$ components of the velocity are equal to zero on the boundary of the obstacles (Dirichlet boundary condition in [1]), or if the single normal component is equal to zero (slip boundary condition in the present case). From a physical view point it shows that the slowing effect of the obstacles is mainly due to the fact that the fluid does not penetrate them (zero normal component of the velocity), rather than to the fact that it sticks to the obstacles because of the viscosity (no-slip or Dirichlet boundary condition). Though the results of the present paper are similar to those of [1], the generalization of their proof is not trivial. Some new technical lemmas are required in order to carry out the machinery of [1]. For example, the extension of the velocity is no longer obvious, and we construct it following an idea of C. Conca (see [11]), D. Cioranescu and J. Saint Jean Paulin (see [10]), and L. Tartar (see [27]). Also, due to the presence of the symmetric stress tensor in the boundary condition, the variational formulation of the Stokes equations is different from that in [1], and its coercivity is proved through a Korntype inequality. Besides recovering the results of [1] for the more restrictive case of a slip boundary condition, we present here a new result concerning the local problem 
in the two-dimensional case. As already pointed out in [1], the 2-D case is completely different from the other ones. We complete our previous study [1] by introducing the precise form of the local problem in the plane. This yields a clearer proof of the paradoxical two-dimensional result which is linked to the well-known Stokes paradox and to the Finn-Smith paradox; see [13]. Finally, the exposition is here more simple (and less general) than in [1]; several technical lemmas are merely quoted, and we refer to [1] for their proof.

We turn now to a brief survey of our main result. We represent solid obstacles in a fluid flow by holes in the fluid domain: $\Omega_{\varepsilon}$ is obtained by removing from a given set $\Omega$, included in $\mathbb{R}^{N}$ a collection of periodically distributed holes $\left(T_{i}^{\varepsilon}\right)_{1 \leqq i \leqq N(\varepsilon)}$ (their number $N(\varepsilon)$ is of order of $\varepsilon^{-N}$ ). Each hole $T_{i}^{\varepsilon}$ is homothetic with ratio $a_{\varepsilon}$ to the same model hole $T$. The hole size $a_{\varepsilon}$ is assumed to be much smaller than the inter-hole distance $\varepsilon$ (i.e., $a_{\varepsilon} / \varepsilon$ goes to zero as $\varepsilon$ does). For a given force $f \in\left[L^{2}(\Omega)\right]^{N}$ and a constant positive viscosity $\mu$, denoting by $u_{c}$ the velocity, and by $p_{c}$ the pressure, the Stokes equations in $\Omega_{c}$ are

$$
\left(S_{\varepsilon}\right)\left\{\begin{array}{ll}
\nabla p_{\varepsilon}-\mu \Delta u_{\varepsilon}=f & \text { in } \Omega_{\varepsilon} \\
\nabla \cdot u_{\varepsilon}=0 & \text { in } \Omega_{\varepsilon}
\end{array} .\right.
$$

We provide the Stokes equations with the so-called slip boundary condition

$$
\text { (BC) } \begin{cases}u_{\varepsilon} \cdot n=0 & \text { on } \partial T_{i}^{\varepsilon} \\ \frac{\alpha}{a_{c}} u_{\varepsilon}=2\left(\frac{\partial u_{\varepsilon}}{\partial n} \cdot n\right) n-\left(\nabla u_{\varepsilon}+{ }^{\nabla} \nabla u_{\varepsilon}\right) n & \text { on } \partial T_{i}^{\varepsilon}, \\ u_{c}=0 & \text { on } \partial \Omega\end{cases}
$$

where the slip coefficient $\alpha$ is a positive constant. The first equation in (BC) expresses that the fluid does not flow through the obstacle $T_{i}^{c}$. The second one is a balance relation between the tangential components of the velocity and the infinitesimal force exerted by the fluid on the obstacle. We define a ratio $\sigma_{\epsilon}$ between the size of the holes and the inter-hole distance

$$
\left\{\begin{array}{lll}
\sigma_{\varepsilon}=\left(\frac{\varepsilon^{N}}{a_{c}^{N-2}}\right)^{1 / 2} & \text { for } & N \geqq 3 \\
\sigma_{\varepsilon}=\varepsilon\left|\log \left(\frac{a_{\varepsilon}}{\varepsilon}\right)\right|^{1 / 2} & \text { for } & N=2
\end{array} .\right.
$$

If the limit of $\sigma_{\varepsilon}$ is strictly positive and finite, then the size of the holes is said to be critical. If the limit is zero (or infinite), then the size is larger (or smaller) than the critical one. These three different limits of $\sigma_{\varepsilon}$ lead to three different homogenized limits for $\left(S_{\varepsilon}\right)$ : a Darcy's law, a Brinkman-type law, and the Stokes equations. More precisely, using the so-called energy method introduced by L. Tartar in [27] and adapted by D. Cioranescu and F. Murat in [9], we prove the following: 
THEOREM 1.1. There exists an extension $\left(E_{\varepsilon} u_{\varepsilon}, P_{\varepsilon}\right)$ of the unique solution $\left(u_{\varepsilon}\right.$, $\left.p_{\varepsilon}\right)$ of $\left(S_{\varepsilon}\right)-(\mathrm{BC})$, with the following properties.

(i) If $\lim _{\varepsilon \rightarrow 0} \sigma_{c}=+\infty$, then $\left(E_{c} u_{\varepsilon}, P_{\varepsilon}\right)$ converges strongly to $(u, p)$ in $\left[H_{0}^{1}(\Omega)\right]^{N} \times\left[L^{2}(\Omega) / \mathbb{R}\right]$, where $(u, p)$ is the unique solution of the Stokes equations

$$
\begin{cases}\nabla p-\mu \Delta u=f & \text { in } \Omega \\ \nabla \cdot u=0 & \text { in } \Omega \\ u=0 & \text { on } \partial \Omega\end{cases}
$$

(In this case the holes are too small, and nothing happens when passing to the limit.)

(ii) If $\lim _{\varepsilon \rightarrow 0} \sigma_{\varepsilon}=\sigma>0$, then $\left(E_{\varepsilon} u_{\varepsilon}, P_{\varepsilon}\right)$ converges weakly to $(u, p)$ in $\left[H_{0}^{1}(\Omega)\right]^{N} \times\left[L^{2}(\Omega) / \mathbb{R}\right]$, where $(u, p)$ is the unique solution of the Brinkman-type law

$$
\begin{cases}\nabla p-\mu \Delta u+\frac{\mu}{\sigma^{2}} M u=f & \text { in } \Omega \\ \nabla \cdot u=0 & \text { in } \Omega \\ u=0 & \text { on } \partial \Omega\end{cases}
$$

(For this critical size of the holes, an additional term appears when passing to the limit.)

(iii) If $\lim _{c \rightarrow 0} \sigma_{\varepsilon}=0$, then $\left(E_{\varepsilon} u_{c} / \sigma_{\varepsilon}^{2}, P_{\varepsilon}\right)$ converges strongly to $(u, p)$ in $\left[L^{2}(\Omega)\right]^{N} \times\left[L^{2}(\Omega) / \mathbb{R}\right]$, where $(u, p)$ is the unique solution of Darcy's law

$$
\begin{cases}u=\frac{M^{-1}}{\mu}(f-\nabla p) & \text { in } \Omega \\ \nabla \cdot u=0 & \text { in } \Omega \\ u \cdot n=0 & \text { on } \partial \Omega\end{cases}
$$

(Thus, if the holes are too large, the Stokes flow degenerates to a Darcy flow when passing to the limit.)

The matrix $M$ appearing in the Brinkman-type law and in the Darcy's law is the same, and it depends only on the model hole $T$ and on the slip coefficient $\alpha$. Moreover, we can compute $M$ thanks to the following

THEOREM 1.2. In the homogenization of the Stokes equations $\left(S_{c}\right)$, for any limit value of $\sigma_{\varepsilon}$, the so-called local problem is 


$$
\left\{\begin{array}{ll}
\nabla q_{k}-\Delta w_{k}=0 & \text { in } \mathbb{R}^{N}-T \\
\nabla \cdot w_{k}=0 & \text { in } \mathbb{R}^{N}-T \\
w_{k} \cdot n=0 & \text { on } \partial T \\
\alpha w_{k}=2\left(\frac{\partial w_{k}}{\partial n} \cdot n\right) n-e\left(w_{k}\right) n & \text { on } \partial T \\
w_{k}=e_{k} & \text { at infinity, for } \quad N \geqq 3 \\
w_{k}(x)=\log |x| e_{k} & \text { at infinity, for } \quad N=2
\end{array},\right.
$$

where $e_{k}$ is the $k$-th unit basis vector in $\mathbb{R}^{N}$. For any dimension $N$, the matrix $M$ is defined in terms of the drag force of the above Stokes flow, i.e.,

$$
M e_{k}=\frac{1}{2^{N}} \int_{\partial T}\left(q_{k} n-e\left(w_{k}\right) n\right),
$$

where $n$ is the normal interior vector of $\partial T$. Furthermore it turns out that

$$
\text { for } N \geqq 3, \quad{ }^{t} e_{k} M e_{i}=\frac{1}{2^{N}}\left[\frac{1}{2} \int_{\mathbb{R}^{N}-T} e\left(w_{k}\right): e\left(w_{i}\right)+\alpha \int_{\partial T} w_{k} \cdot w_{i}\right] ;
$$

for $N=2$, whatever the size and shape of the model hole $T$ and the value of the slip coefficient $\alpha$ are, $M$ is always equal to $\pi I d$.

All other possible scalings of the slip coefficient are examined in Section 3; they yield the same homogenized equations as before. We emphasize that the permeability tensor $M^{-1}$ in the above Darcy's law is completely different from that obtained by the two-scale expansions method when the holes size $a_{\varepsilon}$ is exactly of order $\varepsilon$ (see [2], [15], [19], [25], [26]). However, in a forthcoming paper (see [4]), we shall prove that they coincide in the so-called low volume fraction limit.

We conclude this introduction by referring to $\mathrm{C}$. Conca in [11] and R. Lipton and M. Avellaneda in [20] for the homogenization of the Stokes equations with other types of mixed boundary conditions (different from our slip boundary condition). We also refer to [1], [6], [18], [21], [23], and [24] for the derivation of Brinkman's law through homogenization of the Stokes equations with a Dirichlet boundary condition (see the introduction to [1] for a brief survey of these works). Finally we mention that in [7] A. Brillard has addressed a similar problem for the Laplacian with a mixed boundary condition. For a general introduction to the homogenization theory, one could see [5], [25], and the references therein. The results of the present paper have been announced in [3].

\section{Notation}

Throughout this paper, $C$ denotes various real positive constants which never depend on $\varepsilon$. The duality products between $H_{0}^{1}(\Omega)$ and $H^{-1}(\Omega)$, and between 
$\left[H_{0}^{1}(\Omega)\right]^{N}$ and $\left[H^{-1}(\Omega)\right]^{N}$, are both denoted by $<,>_{H^{-1}, H_{0}^{1}(\Omega)}$. The canonical basis of $\mathbb{R}^{N}$ is denoted by $\left(e_{k}\right)_{1 \leqq k \leqq N}$. If $u(x)$ is a vector-valued function from $\mathbb{R}^{N}$ to $\mathbb{R}^{N}$, then

- The gradient of $u$ is an $N \times N$ tensor: $\nabla u=\left(\partial u_{i} / \partial x_{j}\right)_{1 \leqq i, j \leqq N}$.

- We denote by $e(u)$ the $N \times N$ symmetric tensor defined by: $e(u)=(\nabla u+$ $\left.{ }^{\prime} \nabla u\right)=\left(\partial u_{i} / \partial x_{j}+\partial u_{j} / \partial x_{i}\right)_{1 \leqq i, j \leqq N}$.

- The tensorial product of $u$ by $v$ (two vectors in $\mathbb{R}^{N}$ ) is denoted by: $u \otimes v=$ $\left(u_{i} v_{j}\right)_{1 \leqq i, j \leqq N}$.

- The inner product of two $N \times N$ tensors $A=\left(a_{i j}\right)$ and $B=\left(b_{i j}\right)$ is denoted by:

$$
A: B=\operatorname{tr}\left({ }^{\prime} A B\right)=\sum_{1 \leqq i, j \leqq N} a_{i j} b_{i j}
$$

\section{Formulation of the Problem}

Let $\Omega$ be a bounded, connected, open set in $\mathbb{R}^{N}(N \geqq 2)$, with Lipschitz boundary $\partial \Omega, \Omega$ being locally located on one side of its boundary. Let $\varepsilon$ be a sequence of strictly positive reals which tends to zero. The set $\Omega$ is covered with a regular mesh of size $2 \varepsilon$, each cell being a cube $P_{i}^{\varepsilon}$, identical to $(-\varepsilon,+\varepsilon)^{N}$. At the center of each cube $P_{i}^{\varepsilon}$ entirely included in $\Omega$ we make a hole $T_{i}^{\varepsilon}$ of size $a_{\varepsilon}$. Every hole $T_{i}^{c}$ is similar to the same model obstacle $T$ rescaled to size $a_{\varepsilon}$. We assume that $T$ is a smooth closed set, which contains a small open ball $B_{r_{0}}$ (with strictly positive radius $r_{0}$ ), is strictly included in the unit open ball $B_{1}$, and is such that $\left(B_{1}-T\right)$ is connected. Throughout the present paper, the main assumption is that the size of the holes $a_{c}$ is smaller than the inter-hole distance $\varepsilon$, i.e.,

$$
\lim _{\varepsilon \rightarrow 0} \frac{a_{\varepsilon}}{\varepsilon}=0
$$

In this section, we do not specify the relation between $a_{c}$ and $\varepsilon$, but we define a ratio $\sigma_{\varepsilon}$ between $a_{\varepsilon}$ and what will turn out to be the critical size in Section 3 .

$$
\left\{\begin{array}{lll}
\sigma_{\varepsilon}=\left(\frac{\varepsilon^{N}}{a_{\varepsilon}^{N-2}}\right)^{1 / 2} & \text { for } & N \geqq 3 \\
\sigma_{\varepsilon}=\varepsilon\left|\log \left(\frac{a_{\varepsilon}}{\varepsilon}\right)\right|^{1 / 2} & \text { for } & N=2
\end{array} .\right.
$$

To be precise, if the limit of $\sigma_{\varepsilon}$, as $\varepsilon$ tends to zero, is strictly positive and finite, then the hole size is called critical. An elementary geometrical consideration gives the number of holes

$$
N(\varepsilon)=\frac{|\Omega|}{(2 \varepsilon)^{N}}[1+o(1)]
$$


The open set $\Omega_{\varepsilon}$ is obtained by removing from $\Omega$ all the holes $\left(T_{i}^{c}\right)_{i=1}^{N(\varepsilon)}: \Omega_{c}=\Omega-$ $\cup_{i=1}^{N(c)} T_{i}^{c}$. Because only the cells entirely included in $\Omega$ are perforated, it follows that no hole meets the boundary $\partial \Omega$. Thus $\Omega_{\varepsilon}$ is also a bounded connected open set, with a smooth boundary $\partial \Omega_{c}$. This boundary is made of two disconnected parts $\partial \Omega$ and $\Gamma_{\varepsilon}$

$$
\partial \Omega_{\varepsilon}=\partial \Omega \cup \Gamma_{\varepsilon} \quad \text { with } \quad \Gamma_{\varepsilon}=\bigcup_{i=1}^{N(e)} \partial T_{i}^{c}
$$

The flow of an incompressible viscous fluid in the domain $\Omega_{\varepsilon}$ under the action of an exterior force $f$ is described by the following Stokes equations (see Remark 3.4 for the case of the Navier-Stokes equations)

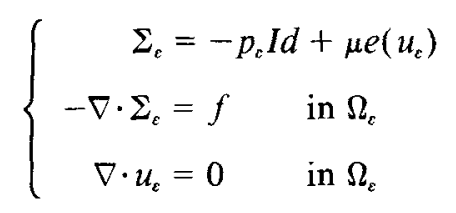

where the stress $\Sigma_{c}$ is an $N \times N$ symmetric tensor, the velocity $u_{\mathrm{\varepsilon}}$ is a vector in $\mathbb{R}^{N}$, the pressure $p_{\varepsilon}$ is a scalar, and the viscosity $\mu$ of the fluid is a strictly positive constant. The Stokes equations (2.5) are endowed with the so-called "slip" boundary condition, which, loosely speaking, allows the fluid to slip on the obstacles, but not to go through them. More precisely, the normal component of the velocity is equal to zero on the obstacles, while the tangential velocity is proportional to the tangential component of the force exerted by the flow on each point of the obstacle's boundary. As is well known in fluid mechanics, this force is equal to the opposite of the normal stress, i.e., to $-\Sigma_{c} n$, where $n$ is the normal vector outward from the fluid domain. Denoting by $\left(\alpha_{\varepsilon} \mu\right)$ a sequence of strictly positive slip coefficients, the slip boundary condition on $\Gamma_{\varepsilon}$ is

$$
\begin{cases}u_{\varepsilon} \cdot n=0 & \text { on } \Gamma_{\varepsilon} \\ \left(\Sigma_{\varepsilon} n+\alpha_{\varepsilon} \mu u_{\varepsilon}\right)=\left({ }^{t} n \Sigma_{\varepsilon} n+\alpha_{\varepsilon} \mu u_{\varepsilon} \cdot n\right) n & \text { on } \Gamma_{\varepsilon}\end{cases}
$$

In the end, as is well known in homogenization, the type of the homogeneous boundary condition on the "large" boundary $\partial \Omega$ is irrelevant; we therefore choose for simplicity a Dirichlet boundary condition

$$
u_{\varepsilon}=0 \quad \text { on } \partial \Omega \text {. }
$$

Regrouping (2.5) to (2.7), and taking into account that $\nabla \cdot u_{\varepsilon}=0$ implies $\nabla \cdot \Sigma_{\varepsilon}=$ $\mu \Delta u_{c}$, we obtain the Stokes system under consideration in this paper 


$$
\begin{cases}\nabla p_{\varepsilon}-\mu \Delta u_{\varepsilon}=f & \text { in } \Omega_{\varepsilon} \\ \nabla \cdot u_{\varepsilon}=0 & \text { in } \Omega_{\varepsilon} \\ u_{\varepsilon} \cdot n=0 & \text { on } \Gamma_{\varepsilon} \\ \alpha_{\varepsilon} u_{\varepsilon}=2\left(\frac{\partial u_{t}}{\partial n} \cdot n\right) n-e\left(u_{\varepsilon}\right) n & \text { on } \Gamma_{\varepsilon} \\ u_{\varepsilon}=0 & \text { on } \partial \Omega\end{cases}
$$

We emphasize that we shall never use the Dirichlet boundary condition on $\partial \Omega$ in the technical lemmas (including Korn's and Poincaré's inequality) which prove the coercivity of this Stokes system. Thus there is no loss of generality in our choice, and all the results of this paper are also valid for any other boundary condition on $\partial \Omega$ (unless otherwise stated).

Now, we define a set $H_{\varepsilon}$ of admissible functions for the velocity

$$
H_{c}=\left\{v \in\left[H^{1}\left(\Omega_{\varepsilon}\right)\right]^{N} / v \cdot n=0 \text { on } \Gamma_{c} \text { and } v=0 \text { on } \partial \Omega\right\}
$$

For a given force $f \in\left[L^{2}(\Omega)\right]^{N}$, the Stokes system (2.8) has the following variational formulation

$$
\left\{\begin{array}{lr}
\text { Find }\left(u_{\varepsilon}, p_{\varepsilon}\right) \in H_{c} \times\left[L^{2}\left(\Omega_{c}\right) / \mathbb{R}\right] & \text { such that } \\
\frac{\mu}{2} \int_{\Omega_{c}} e\left(u_{\varepsilon}\right): e(v)-\int_{\Omega_{c}} p_{\varepsilon} \nabla \cdot v+\mu \alpha_{c} \int_{\Gamma_{c}} u_{c} \cdot v=\int_{\Omega_{c}} f \cdot v \\
\int_{\Omega_{c}} q \nabla \cdot u_{\varepsilon}=0 & \text { for each } v \in H_{\varepsilon}
\end{array}\right.
$$

Remark 2.1. In [1] the Stokes equations (2.5) were considered with a Dirichlet boundary condition on the obstacles

$$
u_{\varepsilon}=0 \quad \text { on } \partial \Omega_{c} .
$$

In that case, because (2.11) does not involve the stress $\Sigma_{\varepsilon}$, the Stokes system (2.5)(2.11) is equivalent to the following variational formulation

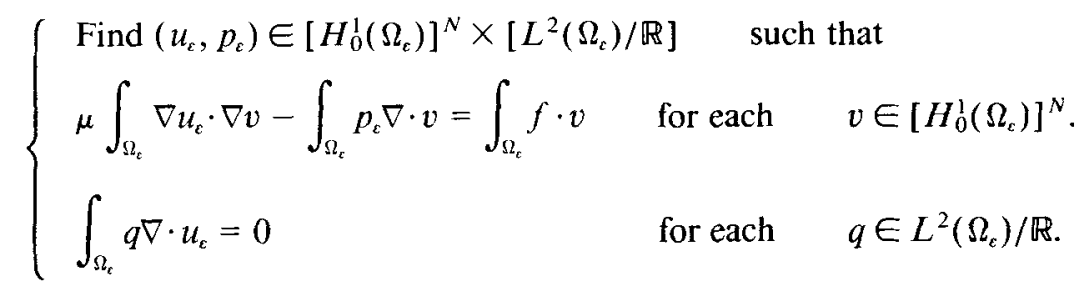


The fundamental difference between (2.10) and (2.12) is the presence in (2.10) of the symmetric stress tensor in the boundary condition. As a consequence, in this paper there are two additional difficulties, beyond those of [1]. First, the extension of the velocity is no longer obvious: in [1] we extended it by zero in the holes, and this extension was continuous because of the Dirichlet boundary condition. Here, we need another kind of extension (see Lemma 2.2). Second, the coercivity of the variational formulation (2.10) (and thus a uniform estimate of the solution) is proved here through a Korn-type inequality (see Lemma 2.4).

PROPOSITION 2.1. If the slip coefficient $\alpha_{e}$ is strictly positive, there exists a unique solution of the variational formulation (2.10), and thus of the equivalent Stokes system (2.8). (Moreover this result does not use the boundary condition on $\partial \Omega$ ).

Proposition 2.1 is proved at the end of this section. Before that we prove several technical lemmas which will also be used in Sections 3 and 4 . We recall that, in the present section, the hole size is not specified. First, let us introduce an extension operator for the velocity.

LEMMA 2.2. There exists a continuous linear map $E_{\varepsilon}$ from $\left[H^{1}\left(\Omega_{c}\right)\right]^{N}$ into $\left[H^{1}(\Omega)\right]^{N}$, such that, for each $v \in\left[H^{1}\left(\Omega_{\varepsilon}\right)\right]^{N}$, we have

$$
\begin{aligned}
& \text { (i) } E_{c} v \equiv v \quad \text { in } \Omega_{c} \\
& \text { (ii) }\left\|e\left(E_{c} v\right)\right\|_{L^{2}(\Omega)} \leqq C\|e(v)\|_{L^{2}(\Omega c)}
\end{aligned}
$$

where $C$ is a positive constant, which depends only on $\Omega$ and $T$, and not on $\varepsilon$.

Furthermore, if $v$ is defined in the whole $\Omega$, i.e., $v \in\left[H^{1}(\Omega)\right]^{N}$, then $e\left(E_{\varepsilon} v\right)$ converges strongly to $e(v)$ in $\left[L^{2}(\Omega)\right]^{N^{2}}$.

Proof: This lemma is classical in the homogenization literature (see Conca [11], Cioranescu and Saint Jean Paulin [10], Tartar [27]), so we only sketch its proof. Each hole $T_{i}^{\varepsilon}$ is included in a ball $B_{i}^{a_{\varepsilon}}$ of radius $a_{\varepsilon}$. We define the closed set $W\left(B_{i}^{a_{c}}-T_{i}^{c}\right)=\left\{v \in\left[H^{1}\left(B_{i}^{a_{\varepsilon}}-T_{i}^{e}\right)\right]^{N} / e(v) \equiv 0\right.$ in $\left.B_{i}^{a_{c}}-T_{i}^{c}\right\}$. It is easy to check that $W\left(B_{i}^{a_{c}}-T_{i}^{\varepsilon}\right)$ is also equal to the set $\left\{v=A x+b\right.$ with $\left.{ }^{t} A=-A\right\}$. Each $v \in$ $\left[H^{1}\left(B_{i}^{a_{t}}-T_{i}^{c}\right)\right]^{N}$ can be decomposed in

$$
v=v_{1}+v_{2} \quad \text { with } \quad v_{1} \in W\left(B_{i}^{a_{c}}-T_{i}^{c}\right) \quad \text { and } \quad v_{2} \in W\left(B_{i}^{a_{c}}-T_{i}^{c}\right)^{\perp} .
$$

The function $v_{1}$ is continuously extended in $T_{i}^{e}$ by its linear value $A x+b$. Now, let $F$ be any continuous extension from $\left[H^{1}\left(B_{1}-T\right)\right]^{N}$ to $\left[H^{1}\left(B_{1}\right)\right]^{N}$. By rescaling $F$, we obtain an extension $F_{i}^{e}$ operating on $\left(B_{i}^{a_{c}}-T_{i}^{c}\right)$. Finally we define the extension $E_{\varepsilon}$ by

$$
\begin{array}{ll}
E_{c} v=v_{1}+F_{i}^{\varepsilon} v_{2} & \text { in each } B_{i}^{a_{\varepsilon}} \\
E_{c} v=v & \text { elsewhere in } \Omega_{c} .
\end{array}
$$


Because $\|v\|_{H^{1}\left(B_{1}\right)}$ and $\|e(v)\|_{L^{2}\left(B_{1}\right)}$ are two equivalent norms in $W\left(B_{1}\right)^{\perp}$, and because the set $B_{1}-T$ is connected, we obtain the desired estimate (2.13) for $E_{\varepsilon}$. Before proving the last statement of Lemma 2.2, let us explain what it means: if $v$ happens to be defined in the whole $\Omega$, then the extension $E_{\varepsilon} v$ is indeed very "close" to $v$ in the sense that the sequence $e\left(E_{\varepsilon} v\right)$ converges strongly to $e(v)$ in $\left[L^{2}(\Omega)\right]^{N^{2}}$, as $\varepsilon$ goes to zero. To see that, we write

$$
\begin{aligned}
\left\|e(v)-e\left(E_{\varepsilon} v\right)\right\|_{L^{2}(\Omega)}^{2} & =\left\|e(v)-e\left(E_{\varepsilon} v\right)\right\|_{L^{2}\left(\Omega-\Omega_{c}\right)}^{2} \\
& \leqq \sum_{i=1}^{N(\varepsilon)}\|e(v)\|_{L^{2}\left(T_{i}^{e}\right)}^{2}+\left\|e\left(E_{\varepsilon} v\right)\right\|_{L^{2}\left(T_{i}^{t}\right)}^{2} \\
& \leqq \sum_{i=1}^{N(c)}\|e(v)\|_{L^{2}\left(T_{i}^{e}\right)}^{2}+C\|e(v)\|_{L^{2}\left(B_{i}^{a_{e}}\right)}^{2} \\
& \leqq C\left\|\chi_{c} e(v)\right\|_{L^{2}(\Omega)}^{2},
\end{aligned}
$$

where $\chi_{\varepsilon}$ is the characteristic function of the set $\cup_{i=1}^{N(c)} B_{i}^{a_{t}}$, which has the property that its measure tends to zero. Then, applying the Lebesgue theorem of dominated convergence, the right-hand side of (2.14) goes to zero with $\varepsilon$, and the lemma is proved.

The next lemma is about a trace result on the boundary $\Gamma_{\varepsilon}$.

LEMMA 2.3. There exists a constant $C$ which does not depend on $\varepsilon$ such that, for any $v \in H^{\mathrm{l}}\left(\Omega_{\varepsilon}\right)$, we have

$$
\frac{\varepsilon^{N}}{a_{\varepsilon}^{N-1}}\|v\|_{L^{2}\left(\Gamma_{\varepsilon}\right)}^{2} \leqq C\left[\|v\|_{L^{2}\left(\Omega_{c}\right)}^{2}+\sigma_{c}^{2}\|\nabla v\|_{L^{2}\left(\Omega_{c}\right)}^{2}\right]
$$

where $\sigma_{\varepsilon}$ is defined in (2.2).

Proof: Let $v \in D\left(\bar{\Omega}_{c}\right)$. As the model hole $T$ is included in the unit ball $B_{1}$, each hole $T_{i}^{\varepsilon}$ is also included in a ball $B_{i}^{a_{\varepsilon}}$ of radius $a_{\varepsilon}$. In a first step, by using radial coordinates, we establish an estimate for the trace of $v$ on $\partial B_{i}^{a_{t}}$. In a second step, we take the trace of $v$ on $\partial T_{i}^{\varepsilon}$, by using a simple trace lemma in $B_{i}^{a_{c}}-T_{i}^{e}$.

Let $B_{i}^{c}$ be the ball of radius $\varepsilon$ with the same center as $B_{i}^{a_{c}}$. Let $r$ be the distance between the center of $B_{i}^{\varepsilon}$ and a point $x \in B_{i}^{c}$, and let $e_{r}$ be the associated unit radial vector. In $B_{i}^{\varepsilon}-B_{i}^{a_{\varepsilon}}$ we have

$$
v(x)-v\left(x+\left(a_{c}-r\right) e_{r}\right)=\int_{a_{c}}^{r} \frac{\partial v}{\partial r}\left[x+(t-r) e_{r}\right] d t
$$


Then

$$
\frac{\varepsilon^{N}}{a_{\varepsilon}^{N-1}}\|v\|_{L^{2}\left(\partial B_{i}^{a_{c}}\right)}^{2} \leqq\|v\|_{L^{2}\left(B_{i}^{c}-B_{i}^{a_{c}}\right)}^{2}+\left\|\left[\int_{a_{\varepsilon}}^{r} \frac{\partial v}{\partial r}\left[x+(t-r) e_{r}\right] d t\right]\right\|_{L^{2}\left(B_{i}^{c}-B_{i}^{a_{i}}\right)}^{2} .
$$

But the Schwarz inequality gives

$$
\left[\int_{a_{c}}^{r} \frac{\partial v}{\partial r}\left[x+(t-r) e_{r}\right] d t\right]^{2} \leqq\left[\int_{a_{c}}^{r}\left[\frac{\partial v}{\partial r}\left[x+(t-r) e_{r}\right]\right]^{2} t^{N-1} d t\right]\left[\int_{a_{c}}^{r} \frac{d t}{t^{N-1}}\right]
$$

Thus

$$
\begin{aligned}
& \left\|\left[\int_{a_{c}}^{r} \frac{\partial v}{\partial r}\left[x+(t-r) e_{r}\right] d t\right]\right\|_{L^{2}\left(B_{i}^{c}-B_{i}^{a_{c}}\right)}^{2} \\
& \leqq\left[\int_{a_{\varepsilon}}^{\varepsilon} \frac{d t}{t^{N-1}}\right] \int_{a_{\varepsilon}}^{c}\left\|\frac{\partial v}{\partial r}\right\|_{L^{2}\left(B_{i}^{c}-B_{i}^{a_{c}}\right)}^{2} r^{N-1} d r \leqq \sigma_{\varepsilon}^{2}\|\nabla v\|_{L^{2}\left(B_{i}^{t}-B_{i}^{a_{e}}\right)}^{2}
\end{aligned}
$$

Hence

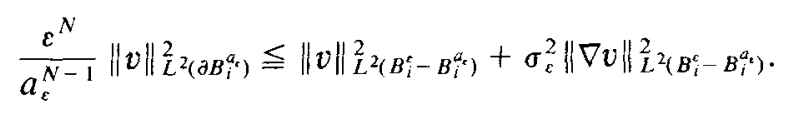

Now, consider the following trace estimate in $B_{1}-T$, which obviously holds true because $B_{1}-T$ is a smooth connected set

$$
\|v\|_{L^{2}(\partial T)} \leqq C\left[\|v\|_{L^{2}\left(\partial B_{1}\right)}+\|\nabla v\|_{I^{2}\left(B_{i}-T\right)}\right]
$$

Rescaling it yields

$$
\|v\|_{L^{2}\left(\partial T_{i}^{\varepsilon}\right)} \leqq C\left[\|v\|_{L^{2}\left(\partial B_{i}^{a^{2}}\right)}+a_{\varepsilon}\|\nabla v\|_{L^{2}\left(B_{i}^{a_{e}}-T_{i}^{\varepsilon}\right)}\right] .
$$

Recalling that $a_{\iota} \ll \varepsilon \ll \sigma_{c}$, and combining the above estimate with (2.15), we obtain

$$
\frac{\varepsilon^{N}}{a_{t}^{N-1}}\|v\|_{L^{2}\left(\partial T_{i}^{\mho}\right)}^{2} \leqq C\left[\|v\|_{L^{2}\left(B_{i}^{t}-B_{i}^{a_{c}}\right)}^{2}+\sigma_{c}^{2}\|\nabla v\|_{L^{2}\left(B_{i}^{c}-T_{i}^{e}\right)}^{2}\right]
$$

Summing (2.16) for $i=1$ to $N(\varepsilon)$ leads to the desired result for any function $v \in$ $D\left(\bar{\Omega}_{c}\right)$. By the density of $D\left(\bar{\Omega}_{\varepsilon}\right)$ in $H^{1}\left(\Omega_{\varepsilon}\right)$, the result holds true also in $H^{1}\left(\Omega_{\varepsilon}\right)$.

The following lemma is concerned with a Korn-type inequality which allows us to prove the coercivity of the variational formulation (see the proof of Proposition 
2.1 below) and to establish an a priori estimate for the velocity in Sections 3 and 4.

LEMMA 2.4. There exists a positive constant $C$, which depends only on $\Omega$ and $T$, such that for each $v \in\left[H^{1}(\Omega)\right]^{N}$

$$
\|\nabla v\|_{L^{2}(\Omega)} \leqq C\left[\left(1+\sigma_{\varepsilon}\right)\|e(v)\|_{L^{2}(\Omega)}+\left[\frac{\varepsilon^{N}}{a_{c}^{N-1}}\right]^{1 / 2}\|v\|_{L^{2}\left(\Gamma_{c}\right)}\right]
$$

Proof: Let $W(\Omega)$ be the closed set $\left\{v \in\left[H^{1}(\Omega)\right]^{N} / e(v) \equiv 0\right.$ in $\left.\Omega\right\}$, which is also equal to $\left\{v=A x+b\right.$ with $\left.{ }^{t} A=-A\right\}$. We denote by $W(\Omega)^{\perp}$ its orthogonal complement with respect to the usual inner product in $\left[H^{1}(\Omega)\right]^{N}$. Each $v \in$ $\left[H^{1}(\Omega)\right]^{N}$ can be decomposed in

$$
v=v_{1}+v_{2} \quad \text { with } \quad v_{1} \in W(\Omega) \quad \text { and } \quad v_{2} \in W(\Omega)^{\perp} .
$$

It is well known that $\|\nabla v\|_{L^{2}(\Omega)}$ and $\|e(v)\|_{L^{2}(\Omega)}$ are two equivalent norms in $W(\Omega)^{\perp}$. Thus, for $v_{2} \in W(\Omega)^{\perp}$, we have

$$
\left\|\nabla v_{2}\right\|_{L^{2}(\Omega)} \leqq C\left\|e\left(v_{2}\right)\right\|_{L^{2}(\Omega)}
$$

where the constant $C$ depends only on $\Omega$. For $v_{1}=(A x+b)$, with ${ }^{t} A=-A$, we compute

$$
\begin{aligned}
\left\|\nabla v_{1}\right\|_{L^{2}(\Omega)}^{2} & =|A|^{2}|\Omega| \\
\left\|v_{1}\right\|_{L^{2}\left(\Gamma_{\varepsilon}\right)}^{2} & =\frac{a_{\varepsilon}^{N-1}|\partial T|}{(2 \varepsilon)^{N}}\|A x+b\|_{L^{2}(\Omega)}^{2}[1+o(1)] .
\end{aligned}
$$

Thus

$$
\left\|\nabla v_{1}\right\|_{L^{2}(\Omega)}^{2} \leqq C \frac{\varepsilon^{N}}{a_{\varepsilon}^{N-1}}\left\|v_{1}\right\|_{L^{2}\left(\Gamma_{\varepsilon}\right)}^{\prime}
$$

where the constant $C$ does not depend on $\varepsilon$, nor on $v_{1}$. Summing (2.17) and (2.18) leads to

$$
\begin{aligned}
\|\nabla v\|_{L^{2}(\Omega)}^{2} & \leqq C\left[\|e(v)\|_{L^{2}(\Omega)}^{2}+\frac{\varepsilon^{N}}{a_{\varepsilon}^{N-1}}\left\|v_{1}\right\|_{L^{2}\left(\Gamma_{\varepsilon}\right)}^{2}\right] \\
& \leqq C\left[\|e(v)\|_{L^{2}(\Omega)}^{2}+\frac{\varepsilon^{N}}{a_{c}^{N-1}}\|v\|_{L^{2}\left(\Gamma_{\varepsilon}\right)}^{2}+\frac{\varepsilon^{N}}{a_{\varepsilon}^{N-1}}\left\|v_{2}\right\|_{L^{2}\left(\Gamma_{\varepsilon}\right)}^{2}\right] .
\end{aligned}
$$


Using the trace Lemma 2.3 we get

$$
\frac{\varepsilon^{N}}{a_{\varepsilon}^{N-1}}\left\|v_{2}\right\|_{L^{2}\left(\Gamma_{\varepsilon}\right)}^{2} \leqq\left\|v_{2}\right\|_{L^{2}(\Omega)}^{2}+\sigma_{\varepsilon}^{2}\left\|\nabla v_{2}\right\|_{L^{2}(\Omega)}^{2} .
$$

Because $v_{2}$ belongs to the orthogonal complement of $W(\Omega)$ (which contains the constants), its average over $\Omega$ is equal to zero. Thus, the Poincaré-Wirtinger inequality gives

$$
\left\|v_{2}\right\|_{L^{2}(\Omega)} \leqq C\left\|\nabla v_{2}\right\|_{L^{2}(\Omega)}
$$

Thanks to (2.17) we deduce from (2.20)

$$
\frac{\varepsilon^{N}}{a_{\varepsilon}^{N-1}}\left\|v_{2}\right\|_{L^{2}\left(\Gamma_{c}\right)}^{2} \leqq\left(1+\sigma_{\varepsilon}^{2}\right)\left\|e\left(v_{2}\right)\right\|_{L^{2}(\Omega)}^{2} .
$$

Plugging the above estimate in (2.19) concludes the proof.

Remark 2.2. Lemmas 2.2, 2.3, and 2.4 do not use any assumptions on the type of the boundary condition on $\Gamma_{c}$ and on $\partial \Omega$. In particular, the fact that the normal component of the velocity is equal to zero on $\Gamma_{\varepsilon}$ is never used. Therefore those lemmas hold true in a more general context. However, Lemma 2.5 below relies upon our choice of the boundary condition on $\Gamma_{\varepsilon}$ for the velocity.

Now, we establish a Poincaré inequality in $H_{\varepsilon}$, which will be used in Sections 3 and 4 for obtaining an a priori estimate for the velocity. that

LEMMA 2.5. There exists a positive constant $C$, which depends only on $\Omega$, such

(i) for each $v \in\left[H^{1}\left(\Omega_{\varepsilon}\right)\right]^{N}$, satisfying $v \cdot n=0$ on $\Gamma_{\varepsilon}$

$$
\|v\|_{L^{2}\left(\Omega_{\epsilon}\right)} \leqq C \sigma_{c}\|\nabla v\|_{L^{2}\left(\Omega_{c}\right)}
$$

(ii) for each $v \in\left[H^{1}(\Omega)\right]^{N}$, satisfying $v \cdot n=0$ on $\Gamma_{c}$

$$
\|v\|_{L^{2}(\Omega)} \leqq C \sigma_{e}\|\nabla v\|_{L^{2}(\Omega)}
$$

Proof: The same arguments as in the previous Lemma 2.3 lead to an inequality similar to (2.15)

$$
\|v\|_{L^{2}\left(B_{i}^{i}-B_{i}^{a_{e}}\right)}^{2} \leqq \frac{\varepsilon^{N}}{a_{c}^{N-1}}\|v\|_{L^{2}\left(\partial B_{i}^{a_{e}}\right)}^{2}+\sigma_{\varepsilon}^{2}\|\nabla v\|_{L^{2}\left(B_{i}^{i}-B_{i}^{a_{e}}\right)}^{2}
$$


Recall that the model hole $T$ contains a ball $B_{r_{0}}$ (with radius $r_{0}>0$ ). Thus, the following Poincaré inequality holds for any $v \in\left[H^{1}\left(B_{1}-T\right)\right]^{N}$ such that $v \cdot n=$ 0 on $\partial T$

$$
\left.\|v\|_{L^{2}\left(B_{1}\right.} \quad T\right) \leqq C\|\nabla v\|_{L^{2}\left(B_{1}-T\right)} .
$$

Besides, we also have the following trace property for any $v \in\left[H^{1}\left(B_{1}-T\right)\right]^{N}$ such that $v \cdot n=0$ on $\partial T$

$$
\|v\|_{L^{2}\left(\partial B_{1}\right)} \leqq C\|\nabla v\|_{L^{2}\left(B_{1}-T\right)}
$$

Rescaling to size $a_{\varepsilon}$ the two previous inequalities give

$$
\begin{aligned}
& \|v\|_{L^{2}\left(B_{i}^{a^{*}}-T_{i}^{\xi}\right)}^{2} \leqq C a_{\varepsilon}^{2}\|\nabla v\|_{L^{2}\left(B_{i}^{a_{-}}-T_{i}^{\tau_{i}}\right)}^{2} \\
& \|v\|_{L^{2}\left(\partial B_{i}^{a_{i}}\right)}^{2} \leqq C a_{\varepsilon}\|\nabla v\|_{L^{2}\left(B_{i}^{a_{c}}-T_{i}^{e}\right)}^{2}
\end{aligned}
$$

for any $v \in\left[H^{1}\left(B_{i}^{a_{\varepsilon}}-T_{i}^{\varepsilon}\right)\right]^{N} \quad$ such that $\quad v \cdot n=0$ on $\partial T_{i}^{e}$.

Recalling that $a_{\varepsilon} \ll \varepsilon \ll \sigma_{\varepsilon}$, and combining (2.23) and (2.24) yields

$$
\|v\|_{L^{2}\left(B_{i}^{c}-T_{i}^{c}\right)}^{2} \leqq C \sigma_{c}^{2}\|\nabla v\|_{L^{2}\left(B_{i}^{c}-Y_{i}^{c}\right)}^{2}
$$

From (2.25), it is not difficult to obtain an equivalent inequality in $P_{i}^{c}-T_{i}^{\varepsilon}$. Then, summing these inequalities for all cells $P_{i}^{\varepsilon}$ gives inequality (2.21). In order to obtain (2.22), we just need to add to the above ingredients the following Poincare inequality in $T$, which holds for any $v \in\left[H^{1}(T)\right]^{N}$ such that $v \cdot n=0$ on $\partial T$

$$
\|v\|_{L^{2}(T)} \leqq\|\nabla v\|_{L^{2}(T)} .
$$

Rescaling it to size $a_{\varepsilon}$, and adding it to (2.25) gives (2.22).

We recall, without proofs, two crucial, but rather technical, results from [1] which are concerned with the extension of the pressure to the whole domain $\Omega$. First, we define a restriction operator $R_{\varepsilon}$ from $\left[H_{0}^{1}(\Omega)\right]^{N}$ into $\left[H_{0}^{1}\left(\Omega_{\varepsilon}\right)\right]^{N}$. Then, following an idea of $\mathrm{L}$. Tartar (see [26]), we extend the gradient of the pressure, by duality in $\left[H^{-1}(\Omega)\right]^{N}$.

LEMMA 2.6. There exists a linear map $R_{\varepsilon}$ operating from $\left[H_{0}^{1}(\Omega)\right]^{N}$ into $\left[H_{0}^{1}\left(\Omega_{\varepsilon}\right)\right]^{N}$ such that

(i) $u \in\left[H_{0}^{1}\left(\Omega_{\varepsilon}\right) \cap H_{0}^{1}(\Omega)\right]^{N} \Rightarrow R_{c} u \equiv u$ in $\Omega_{\varepsilon}$

(ii) $\nabla \cdot u=0 \quad$ in $\Omega \Rightarrow \nabla \cdot\left(R_{\varepsilon} u\right)=0 \quad$ in $\Omega_{\varepsilon}$

(iii) $\left\|\nabla\left(R_{\varepsilon} u\right)\right\|_{L^{2}\left(\Omega_{c}\right)} \leqq C\left[\|\nabla u\|_{L^{2}(\Omega)}+\left(1 / \sigma_{\varepsilon}\right)\|u\|_{L^{2}(\Omega)}\right]$ and $C$ does not depend on $\varepsilon$. 
LEMMA 2.7. Let $p_{\varepsilon}$ be a function in $L^{2}\left(\Omega_{\varepsilon}\right) / \mathbb{R}$. There exists a unique function $P_{\varepsilon} \in L^{2}(\Omega) / \mathbb{R}$ which satisfies the following equality

$$
\left\langle\nabla P_{c}, v\right\rangle_{H^{-1}, H_{0}^{1}(\Omega)}=\left\langle\nabla p_{\varepsilon}, R_{\varepsilon} v\right\rangle_{H^{-1}, H_{0}^{1}\left(\Omega_{\varepsilon}\right)} \quad \text { for each } \quad v \in\left[H_{0}^{1}(\Omega)\right]^{N} .
$$

Thanks to Lemma 2.6, $P_{\varepsilon}$ is an extension of $p_{c}$, but in addition, because of the explicit construction of $R_{c}$, we obtain the precise value of $P_{\varepsilon}$ in the holes

$$
P_{\varepsilon}=p_{\varepsilon} \quad \text { in } \Omega_{\varepsilon} \quad \text { and } \quad P_{\varepsilon}=\frac{1}{\left|C_{i}^{\varepsilon}\right|} \int_{C_{i}^{\varepsilon}} p_{\varepsilon} \quad \text { in each hole } T_{i}^{c}
$$

where $C_{i}^{e}$ is a control volume around the hole $T_{i}^{c}$, defined as the part outside $T_{i}^{e}$ of the ball of radius $\varepsilon$ with same center as $T_{i}^{e}$.

(See Section 2.2 and Proposition 3.4.10 in [1] for proofs of Lemmas 2.6 and 2.7.)

Proof of Proposition 2.1: Thanks to the Korn-type inequality obtained in Lemma 2.4, the variational formulation $(2.10)$ is coercive as soon as the slip coefficient $\alpha_{\varepsilon}$ is strictly positive

$$
K_{\varepsilon}\left\|\nabla u_{\varepsilon}\right\|_{L^{2}\left(\Omega_{\varepsilon}\right)}^{2} \leqq K_{\varepsilon}\left\|\nabla\left(E_{c} u_{\varepsilon}\right)\right\|_{L^{2}(\Omega)}^{2} \leqq C\left[\left\|e\left(u_{\varepsilon}\right)\right\|_{L^{2}\left(\Omega_{\varepsilon}\right)}^{2}+\alpha_{\varepsilon}\left\|u_{e}\right\|_{L^{2}\left(\Gamma_{\varepsilon}\right)}^{2}\right]
$$

with

$$
K_{\varepsilon}=\min \left[\frac{1}{1+\sigma_{\varepsilon}^{2}}, \frac{\alpha_{\varepsilon} a_{\varepsilon}^{N-1}}{\varepsilon^{N}}\right] .
$$

Then a variant of the Lax-Milgram theorem asserts that the variational formulation (2.10) has a unique solution (see, e.g., Girault Raviart [14]). Finally, integrating by parts in (2.10) it is easy to show that a solution of $(2.10)$ is also a weak solution of the Stokes system (2.8), and vice-versa.

\section{The Critical Scaling: Main Results}

In this section we prove the convergence of the homogenization process, when the hole size and the slip coefficient scale as follows:

$$
\left\{\begin{array}{c}
\lim _{\varepsilon \rightarrow 0} \alpha_{\varepsilon} a_{c}=\alpha \\
\lim _{\varepsilon \rightarrow 0} \sigma_{\varepsilon}=\sigma,
\end{array}\right.
$$

where $\alpha$ and $\sigma$ are strictly positive constants. In the sequel, we will refer to those relations (3.1) as the critical scaling. For example, the latter relation in (3.1) is 
satisfied by a hole size $a_{\varepsilon}$ equal to $C_{0} \varepsilon^{N /(N-2)}$ for $N \geqq 3$, or $\exp \left\{-C_{0} / \varepsilon^{2}\right\}$ for $N=$ 2 with $C_{0}>0$, and $\sigma$ given by $\sigma=1 / C_{0}^{(N-2) / 2}$ for $N \geqq 3$, or $\sigma=C_{0}^{1 / 2}$ for $N=2$. Using the lemmas of the first section, we obtain some a priori estimates for the solution of the variational formulation (2.10), without making essential use of the Dirichlet boundary condition on the "large" boundary $\partial \Omega$.

Proposition 3.1. Let $\left(u_{c}, p_{c}\right)$ be the solution of $(2.10)$, and $\left(E_{\varepsilon}\left(u_{c}\right), P_{\varepsilon}\right)$ its extension in $\Omega$, as defined in Lemmas 2.2 and 2.7. Then we have

$$
\begin{array}{r}
\left\|E_{\varepsilon} u_{\varepsilon}\right\|_{L^{2}(\Omega)}+\left\|\nabla\left(E_{\varepsilon} u_{\varepsilon}\right)\right\|_{L^{2}(\Omega)} \leqq C\|f\|_{L^{2}(\Omega)} \\
\left\|P_{\varepsilon}\right\|_{L^{2}(\Omega) / R} \leqq C\|f\|_{L^{2}(\Omega)}
\end{array}
$$

where the constant $C$ depends only on $\Omega$ and $T$ (and not on $\varepsilon$ ).

Proof: Introducing $v=u_{\varepsilon}$ in (2.10) leads to

$$
\frac{\mu}{2} \int_{\Omega_{\varepsilon}}\left|e\left(u_{c}\right)\right|^{2}+\mu \alpha_{\varepsilon} \int_{\Gamma_{\varepsilon}}\left|u_{\varepsilon}\right|^{2}=\int_{\Omega_{\varepsilon}} f \cdot u_{\varepsilon}
$$

Because $u_{\varepsilon} \in\left[H^{1}\left(\Omega_{\varepsilon}\right)\right]^{N}$ and satisfies $u_{t} \cdot n=0$ on $\Gamma_{\varepsilon}$, Poincaré's inequality (2.21) (see Lemma 2.5) furnishes an upper bound of the right-hand side of (3.2)

$$
\left|\int_{\Omega_{\varepsilon}} f \cdot u_{c}\right| \leqq C\|f\|_{L^{2}(\Omega)}\left\|\nabla u_{c}\right\|_{L^{2}\left(\Omega_{\varepsilon}\right)} \leqq\|f\|_{L^{2}(\Omega)}\left\|\nabla\left(E_{\varepsilon} u_{c}\right)\right\|_{L^{2}(\Omega)} .
$$

Thanks to the properties of the extension $E_{\varepsilon}$ and to the scaling (3.1), the Korntype inequality of Lemma 2.4 gives a lower bound of the left-hand side of (3.2)

$$
\begin{aligned}
\frac{\mu}{2} \int_{\Omega_{\varepsilon}}\left|e\left(u_{\varepsilon}\right)\right|^{2} & +\mu \alpha_{\varepsilon} \int_{\Gamma_{\varepsilon}}\left|u_{\varepsilon}\right|^{2} \\
& \geqq C\left[\int_{\Omega}\left|e\left(E_{\varepsilon} u_{c}\right)\right|^{2}+\frac{\alpha}{a_{\varepsilon}} \int_{\Gamma_{c}}\left|E_{c}\left(u_{\varepsilon}\right)\right|^{2}\right] \geqq C\left\|\nabla\left(E_{c} u_{\varepsilon}\right)\right\|_{L^{2}(\Omega)}^{2} .
\end{aligned}
$$

Finally, (3.3) and (3.4) yield

$$
\left\|\nabla\left(E_{\varepsilon} u_{\varepsilon}\right)\right\|_{L^{2}(\Omega)} \leqq C\|f\|_{L^{2}(\Omega)},
$$

and Poincaré's inequality (2.22) gives

$$
\left\|\mathrm{E}_{\varepsilon} u_{\varepsilon}\right\|_{L^{2}(\Omega)} \leqq C\|f\|_{L^{2}(\Omega)}
$$


Now we turn to an estimate for the pressure. Recall the definition (2.26) of the extension $P_{\varepsilon}$

$$
\left\langle\nabla P_{c}, v\right\rangle_{H^{-1}, H_{0}^{\mathrm{l}}(\Omega)}=\left\langle\nabla p_{c}, R_{c} v\right\rangle_{H^{-1}, H_{0}^{1}\left(\Omega_{c}\right)} \quad \text { for each } \quad v \in\left[H_{0}^{1}(\Omega)\right]^{N} .
$$

Introducing the Stokes equation, and integrating by parts, leads to

$$
\left|\left\langle\nabla P_{\varepsilon}, v\right\rangle\right| \leqq\left\|\nabla u_{\varepsilon}\right\|_{L^{2}\left(\Omega_{\varepsilon}\right)}\left\|\nabla\left(R_{\varepsilon} v\right)\right\|_{L^{2}\left(\Omega_{\varepsilon}\right)}+\|f\|_{L^{2}(\Omega)}\left\|R_{\varepsilon} v\right\|_{L^{2}\left(\Omega_{\varepsilon}\right)} .
$$

Thanks to the properties of $R_{\varepsilon}$ in Lemma 2.6, and to the previous estimate on the velocity $u_{\varepsilon}$, we obtain

$$
\left\|\nabla P_{c}\right\|_{H^{-1}(\Omega)} \leqq C\|f\|_{L^{2}(\Omega)}
$$

But a well-known lemma of functional analysis claims that, because of (3.5), $P_{\varepsilon}$ belongs to $L^{2}(\Omega)$, and we have

$$
\left\|P_{c}\right\|_{L^{2}(\Omega) / \mathbb{R}} \leqq C(\Omega)\left\|\nabla P_{c}\right\|_{H^{-1}(\Omega)}
$$

Hence (3.5) gives the result.

Because of the estimates in Proposition 3.1, one can extract a subsequence of extensions of the solutions, still denoted $\left(E_{\varepsilon} u_{\varepsilon}, P_{c}\right)_{\varepsilon>0}$ and there exists some $(u$, $p) \in\left[H_{0}^{1}(\Omega)\right]^{N} \times L^{2}(\Omega) / \mathbb{R}$ such that $\left(E_{\varepsilon} u_{\varepsilon}, P_{\varepsilon}\right)$ converges weakly to $(u, p)$ in $\left[H_{0}^{1}(\Omega)\right]^{N} \times L^{2}(\Omega) / \mathbb{R}$. The problem is now to find which equation is satisfied by the limit (or homogenized) velocity $u$ and pressure $p$. For that purpose we use the energy method introduced by L. Tartar in [27] (see also F. Murat [22]). The main idea is to compute in a unit cell the solutions of the "local" problem corresponding to the Stokes system (2.8). Then, by rescaling and extending by periodicity these local solutions, we construct so-called test functions (which can be interpreted as boundary layers around the holes $T_{i}^{\varepsilon}$ ). After multiplication by a fixed smooth function, they are introduced in the variational formulation. Finally, integrating by parts, we can pass to the limit in the variational formulation, and obtain the limit equation. The following lemma gives the so-called local problem.

LEMMA 3.2. For $N \geqq 3$ the local problem is

$$
\left\{\begin{array}{rlrl}
\nabla q_{k}-\Delta w_{k} & =0 & & \text { in } \mathbb{R}^{N}-T \\
\nabla \cdot w_{k} & =0 & & \text { in } \mathbb{R}^{N}-T \\
w_{k} \cdot n & =0 & & \text { on } \partial T \\
\alpha w_{k} & =2\left(\frac{\partial w_{k}}{\partial n} \cdot n\right) n-e\left(w_{k}\right) n \quad \text { on } \partial T \\
w_{k} & =e_{k} \quad & \text { at } \infty .
\end{array}\right.
$$


There exists a unique solution of (3.6) such that $\left\|q_{k}\right\|_{L^{2}\left(\mathbb{R}^{N}-T\right)}<+\infty$ and $\left\|\nabla w_{k}\right\|_{L^{2}\left(\mathbb{R}^{N}-T\right)}<+\infty$. Moreover, denoting by $F_{k}$ the drag force applied on $T$, i.e., $F_{k}=\int_{\partial T}\left(q_{k} n-e\left(w_{k}\right) n\right)$, where $n$ is the interior normal vector of $\partial T$, we have

$$
F_{k} \cdot e_{i}=\left[\frac{1}{2} \int_{\mathbb{R}^{N}-T} e\left(w_{k}\right) \cdot e\left(w_{i}\right)+\alpha \int_{\partial T} w_{k} \cdot w_{i}\right] \text { for each } i \in[1 ; N]
$$

For $N=2$ the local problem is

$$
\left\{\begin{array}{rlrl}
\nabla q_{k}-\Delta w_{k} & =0 \quad & \text { in } \mathbb{R}^{N}-T \\
\nabla \cdot w_{k} & =0 \quad \text { in } \mathbb{R}^{N}-T \\
w_{k} \cdot n & =0 \quad \text { on } \partial T \\
\alpha w_{k} & =2\left(\frac{\partial w_{k}}{\partial n} \cdot n\right) n-e\left(w_{k}\right) n \quad \text { on } \partial T \\
w_{k} & =(\log r) e_{k} \quad \text { at } \infty .
\end{array}\right.
$$

There exists a unique solution of (3.8), and still denoting by $F_{k}=\int_{\partial T}\left(q_{k} n-\right.$ $\left.e\left(w_{k}\right) n\right)$ the drag force applied on $T$, we obtain a paradoxical result: whatever the size and the shape of $T$, and for any value of the slip coefficient $\alpha$, we have

$$
F_{k}=4 \pi e_{k} \quad \text { for } \quad k=1,2 .
$$

(See Section 5 for the proof.)

DEFINITION 3.1. Let us define a matrix $M$ by

$$
M e_{k}=\frac{1}{2^{N}} F_{k}
$$

where $F_{k}$ is the drag force introduced in the above Lemma 3.2. In the two-dimensional case, we always have $M=\pi I d$, but in the other cases $(N \geqq 3)$, the matrix $M$ is given by formula (3.7) and depends on the slip coefficient $\alpha$. In any case, $M$ is a symmetric positive definite matrix.

Remark 3.1. There are two fundamental differences between the local problem in two and three (or more) dimensions. First, the profile of the velocity at infinity is uniform for the three (or more) dimensional case, while it is logarithmic for the two-dimensional case. Actually the celebrated Stokes paradox asserts that, in the plane, there is no solution of (3.8) which remains bounded at infinity, except the zero solution (see, e.g., Finn [12]). That is why there is logarithmic growth at infinity in (3.8). Second, although in the three (or more) dimensional case the drag force $F_{k}$ heavily depends both on the size and shape of the obstacle $T$, and on the slip coefficient $\alpha$, in two dimensions it does not, and actually is equal to the 
drag force obtained with a Dirichlet boundary condition. This paradox has already been pointed out by R. Finn and D. Smith in [13].

PROPOSITION 3.3. With the help of the local solutions $\left(w_{k}, q_{k}\right)_{1 \leqq k \leqq N}$, we can construct test functions $\left(w_{k}^{\varepsilon}, q_{k}^{c}\right)$, which satisfy the following properties:

(i) $q_{k}^{\varepsilon} \rightarrow 0$ in $L^{2}(\Omega) / \mathbb{R}$ weakly.

(ii) $w_{k}^{\varepsilon} \rightarrow e_{k}$ in $\left[H^{1}(\Omega)\right]^{N}$ weakly, and $\nabla \cdot w_{k}^{\varepsilon}=0$ in $\Omega$.

(iii) $w_{k}^{c} \cdot n=0$ and $\left(\alpha / a_{e}\right) w_{k}^{c}=2\left[\left(\partial w_{k}^{c} / \partial n\right) \cdot n\right] n-e\left(w_{k}^{c}\right) n$ on $\Gamma_{\varepsilon}$.

(iv) Let $\mu_{k}^{\varepsilon}=\nabla q_{k}^{\varepsilon}-\Delta w_{k}^{\varepsilon} \in\left[H^{-1}\left(\Omega_{\varepsilon}\right)\right]^{N}$. Then $\mu_{k}^{\varepsilon}$ has compact support in $\Omega_{c}$.

Moreover, extending $\mu_{k}^{c}$ by 0 in $\Omega-\Omega_{c}$ we have

$$
\mu_{k}^{e} \rightarrow \frac{1}{\sigma^{2}} M e_{k} \quad \text { in } \quad\left[H^{-1}(\Omega)\right]^{N} \quad \text { strongly. }
$$

Remark 3.2. Let us explain in more detail property (iv) of Proposition 2.5. A priori the distribution ( $\nabla q_{k}^{\varepsilon}-\Delta w_{k}^{\varepsilon}$ ) is defined in the whole of $\Omega$; because of (i) and (ii) it is bounded (but not necessarily compact) in $\left[H^{-1}(\Omega)\right]^{N}$, and its weak limit is zero. But if we restrict it to $\left[H^{-1}\left(\Omega_{\varepsilon}\right)\right]^{N}$, that is, if we skip the contributions coming from the obstacles and their boundaries, then it becomes a compact sequence with a non-zero limit. From a technical view point, in the process of extending $\mu_{k}^{\varepsilon}$ by 0 in $\Omega-\Omega_{\varepsilon}$, the main ingredient is that it has compact support in $\Omega_{\varepsilon}$, i.e., it is equal to zero in a vicinity of the boundary $\partial \Omega_{\varepsilon}$.

Proposition 3.3 is proved in Section 5. Let us explain roughly what is the connection between Lemma 3.2 and Proposition 3.3. The test functions $\left(w_{k}^{c}, q_{k}^{c}\right)_{1 \leqq k \leqq N}$ are obtained by rescaling the local solutions $\left(w_{k}, q_{k}\right)_{1 \leqq k \leqq N}$ in each cell $P_{i}^{c}$. Let $C_{i}^{\prime c}$ be the ball of radius $\varepsilon / 2$ whose center coincides with that of $P_{i}^{c}$. In $C_{i}^{\prime \varepsilon}$ we define

$$
\left\{\begin{array}{l}
w_{k}^{\varepsilon}(x)=w_{k}\left(\frac{x}{a_{\varepsilon}}\right) \text { and } q_{k}^{\varepsilon}=\frac{1}{a_{\varepsilon}} q_{k}\left(\frac{x}{a_{\varepsilon}}\right) \text { for } N \geqq 3 \\
w_{k}^{\varepsilon}(x)=\frac{1}{\log \frac{\varepsilon}{2 a_{\varepsilon}}} w_{k}\left(\frac{x}{a_{\varepsilon}}\right) \text { and } q_{k}^{\varepsilon}=\frac{1}{a_{\varepsilon} \log \left(\frac{\varepsilon}{2 a_{\varepsilon}}\right)} q_{k}\left(\frac{x}{a_{\varepsilon}}\right) \text { for } N=2
\end{array}\right.
$$

In the remaining part $P_{i}^{\varepsilon}-C_{i}^{\prime \varepsilon}$ of each cell, we match the above expression with the value $w_{k}^{c}=e_{k}$ on the boundary $\partial P_{i}^{c}$, in order to insure that $w_{k}^{c}$ belongs to $\left[H^{1}(\Omega)\right]^{N}$.

We are now in a position to state our main result

THEOREM 3.4. Let $M$ be the matrix defined in Definition 3.1. Let $\left(u_{\varepsilon}, p_{c}\right)$ be the unique solution of the Stokes system (2.8). Then its extension $\left(E_{\varepsilon} u_{\varepsilon}, P_{\varepsilon}\right)$ converges 
weakly in $\left[H_{0}^{1}(\Omega)\right]^{N} \times\left[L^{2}(\Omega) / \mathbb{R}\right]$ to $(u, p)$, which is the unique solution of the homogenized system $(3.10)$

$$
\left\{\begin{array}{l}
\text { Find }(u, p) \in\left[H_{0}^{1}(\Omega)\right]^{N} \times\left[L^{2}(\Omega) / \mathbb{R}\right] \quad \text { such that: } \\
\nabla p-\mu \Delta u+\frac{\mu}{\sigma^{2}} M u=f \quad \text { in } \Omega \\
\nabla \cdot u=0 \quad \text { in } \Omega
\end{array}\right.
$$

Remark 3.3. The homogenized system (3.10) is a law of Brinkman type (see the original paper of Brinkman [8]). The new term $M u$ in (3.10) expresses the presence of the holes which have otherwise disappeared after passing to the limit. We already obtained in [1] a Brinkman type law as the limit system of the Stokes equations with a Dirichlet boundary condition. Although the size of the holes is the same in both cases, the local problem and therefore the matrix $M$ are different, except in two dimensions. The dependence of the matrix $M$ on $\alpha$ is not obvious. However, we can compare the value of $M$ for two different values of $\alpha$

$$
0<\alpha_{1} \leqq \alpha_{2} \Rightarrow{ }^{t} \xi M\left(\alpha_{1}\right) \xi \leqq{ }^{t} \xi M\left(\alpha_{2}\right) \xi \quad \text { for any } \quad \xi \in \mathbb{R}^{N}
$$

Moreover, the limit of $M(\alpha)$, when $\alpha$ tends to infinity, is the matrix $M$ obtained with a Dirichlet boundary condition (see Section 5). Finally, in order to illustrate the dependence of $\alpha$ on $M$, we give its value when the obstacle $T$ is the unit ball

$$
M=\frac{S_{N}}{2^{N}} \frac{N(N-2)}{N-1} \frac{2+\alpha}{N+\alpha} I d \quad \text { for } \quad N \geqq 3
$$

( $S_{n}$ is the area of the unit sphere in $\mathbb{R}^{N}$ ).

Proof: We apply the energy method (introduced by L. Tartar in [27], and adapted to the case of domains perforated with tiny holes by $D$. Cioranescu and $F$. Murat in [9]). For any fixed $\phi \in D(\Omega)$, we introduce in the variational formulation (2.10) the test functions

$$
\left(\phi W_{k}^{\varepsilon}\right) \in H_{\varepsilon} \quad \text { and } \quad\left(\phi q_{k}^{\varepsilon}\right) \in L^{2}\left(\Omega_{\varepsilon}\right) / \mathbb{R}
$$

We obtain

$$
\left\{\begin{array}{l}
\frac{\mu}{2} \int_{\Omega_{\varepsilon}} e\left(u_{\varepsilon}\right): e\left(\phi w_{k}^{e}\right)-\int_{\Omega_{c}} p_{c} \nabla \cdot\left(\phi w_{k}^{\varepsilon}\right)+\mu \alpha_{\varepsilon} \int_{\Gamma_{\varepsilon}} u_{c} \cdot\left(\phi w_{k}^{c}\right)=\int_{\Omega_{c}} f \cdot\left(\phi w_{k}^{\varepsilon}\right) \\
\int_{\Omega_{c}}\left(\phi q_{k}^{\varepsilon}\right) \nabla \cdot u_{c}=0
\end{array}\right.
$$


624

G. ALLAIRE

Expanding (3.11), and using that $w_{k}^{e}$ is divergence-free, gives

(3.12) $\left\{\begin{array}{l}\frac{\mu}{2} \int_{\Omega_{c}} \phi e\left(u_{c}\right): e\left(w_{k}^{c}\right)+\frac{\mu}{2} \int_{\Omega_{e}} e\left(u_{\varepsilon}\right):\left(\nabla \phi \circledast w_{k}^{c}+w_{k}^{c} \circledast \nabla \phi\right) \\ \quad-\int_{\Omega_{c}} p_{\varepsilon} w_{k}^{c} \cdot \nabla \phi+\mu \alpha_{\varepsilon} \int_{\Gamma_{c}} \phi u_{\varepsilon} \cdot w_{k}^{c}=\int_{\Omega_{c}} \phi f \cdot w_{k}^{c} \\ \int_{\Omega_{c}} \phi q_{k}^{c} \nabla \cdot u_{c}=0 .\end{array}\right.$

We integrate by parts in the first equation of (3.12)

$$
\begin{aligned}
\int_{\Omega_{c}} \phi e\left(u_{\varepsilon}\right): e\left(w l_{k}^{\varepsilon}\right)=\int_{\Omega_{c}} e\left(\phi u_{\varepsilon}\right): e\left(w_{k}^{\varepsilon}\right) & \\
& \quad-\int_{\Omega_{\varepsilon}} e\left(w_{k}^{c}\right):\left(\nabla \phi \otimes u_{c}+u_{c} \otimes \nabla \phi\right),
\end{aligned}
$$

and

(3.14) $\frac{\mu}{2} \int_{\Omega_{c}} e\left(\phi u_{c}\right): e\left(w_{k}^{c}\right)=-\mu \int_{\Omega_{c}} \phi u_{c} \cdot \Delta w_{k}^{c}+\mu \int_{\Gamma_{t}} \phi u_{c} \cdot\left(\frac{\partial w_{k}^{c}}{\partial n}+{ }^{\prime} \nabla w_{k}^{c} n\right)$.

An integration by parts in the second equation of (3.12) gives

$$
\int_{\Omega_{c}} \phi q_{k}^{\varepsilon} \nabla \cdot u_{\varepsilon}=-\int_{\Omega_{c}} \phi u_{c} \cdot \nabla q_{k}^{\varepsilon}-\int_{\Omega_{c}} q_{k}^{\varepsilon} u_{\varepsilon} \cdot \nabla \phi+\int_{\Gamma_{\varepsilon}} \phi q_{k}^{\varepsilon} u_{\varepsilon} \cdot n
$$

Summing the two equations in (3.12), and combining (3.13) with (3.15) yields

$$
\begin{aligned}
& \mu \int_{\Omega_{\varepsilon}} \phi u_{c} \cdot\left(\nabla q_{k}^{c}-\Delta w_{k}^{c}\right)+\frac{\mu}{2} \int_{\Omega_{c}} e\left(u_{\varepsilon}\right):\left(\nabla \phi \otimes w_{k}^{c}+w_{k}^{c} \otimes \nabla \phi\right) \\
& -\frac{\mu}{2} \int_{\Omega_{\varepsilon}} e\left(w_{k}^{\varepsilon}\right):\left(\nabla \phi \otimes u_{c}+u_{c} \otimes \nabla \phi\right)+\mu \int_{\Omega_{\varepsilon}} q_{k}^{\varepsilon} u_{c} \cdot \nabla \phi \\
& -\int_{\Omega_{\varepsilon}} p_{\varepsilon} w_{k}^{c} \cdot \nabla \phi+\mu \int_{\Gamma_{\varepsilon}} \phi u_{\varepsilon} \cdot\left(\frac{\partial w_{k}^{c}}{\partial n}+{ }^{t} \nabla w_{k}^{c} n-q_{k}^{c} n+\alpha_{\varepsilon} w_{k}^{\varepsilon}\right)=\int_{\Omega_{\varepsilon}} \phi f \cdot w_{k}^{\varepsilon} .
\end{aligned}
$$

Denote by $1_{\Omega_{c}}$ the characteristic function of the set $\Omega_{\varepsilon}$, which is equal to 1 in $\Omega_{\varepsilon}$ and 0 elsewhere. Introducing the extensions of $\left(u_{c}, p_{\varepsilon}\right)$, and using the properties of $\left(w_{k}^{c}, q_{k}^{c}\right)$ (see Proposition 3.3), we obtain from (3.16) 


$$
\begin{aligned}
& \mu\left\langle\mu_{k}^{\varepsilon}, \phi E_{\varepsilon} u_{c}\right\rangle_{H^{-1}, H_{0}^{1}(\Omega)}+\frac{\mu}{2} \int_{\Omega} 1_{\Omega_{c}} e\left(E_{c} u_{c}\right):\left(\nabla \phi \otimes w_{k}^{c}+w_{k}^{\varepsilon} \otimes \nabla \phi\right) \\
& -\frac{\mu}{2} \int_{\Omega} 1_{\Omega_{c}} e\left(w_{k}^{e}\right):\left(\nabla \phi \otimes E_{\varepsilon} u_{c}+E_{\varepsilon} u_{c} \otimes \nabla \phi\right)+\mu\left(\alpha_{c}-\frac{\alpha}{a_{\varepsilon}}\right) \int_{\Gamma_{\varepsilon}} \phi u_{\varepsilon} \cdot w_{k}^{c} \\
& +\mu \int_{\Omega} 1_{\Omega_{\varepsilon}} q_{k}^{\varepsilon} E_{c}\left(u_{\varepsilon}\right) \cdot \nabla \phi-\int_{\Omega} 1_{\Omega_{c}} P_{c} w_{k}^{\varepsilon} \cdot \nabla \phi=\int_{\Omega} 1_{\Omega_{c}} \phi f \cdot w_{k}^{\varepsilon} .
\end{aligned}
$$

In order to pass to the limit in (3.17), we note that, if a sequence $v_{c}$ converges strongly to a limit $v$ in $L^{2}(\Omega)$, then the sequence $1_{\Omega_{c}} v_{c}$ converges strongly to the same limit $v$ in $L^{2}(\Omega)$ (because the measure of $\Omega-\Omega_{\varepsilon}$ tends to zero). Thus, using the estimates of Propositions 3.1 and 3.3, and Rellich's theorem, we obtain

$$
\begin{aligned}
\frac{\mu}{\sigma^{2}}\left\langle M e_{k}, \phi u\right\rangle_{H^{-1}, H_{0}^{1}(\Omega)}+\frac{\mu}{2} \int_{\Omega} e(u):\left(\nabla \phi \otimes e_{k}\right. & \left.+e_{k} \otimes \nabla \phi\right) \\
& -\int_{\Omega} p e_{k} \cdot \nabla \phi=\int_{\Omega} \phi f \cdot e_{k} .
\end{aligned}
$$

Integrating (3.18) by parts, and using the symmetry of $M$, gives

$$
\frac{\mu}{\sigma^{2}}\left\langle M u, \phi e_{k}\right\rangle-\mu\left\langle\Delta u, \phi e_{k}\right\rangle+\left\langle\nabla p, \phi e_{k}\right\rangle=\left\langle f, \phi e_{k}\right\rangle \text { for each } k \in[1 ; N]
$$

Thus

$$
\nabla p-\mu \Delta u+\frac{\mu}{\sigma^{2}} M u=f \quad \text { in }\left[D^{\prime}(\Omega)\right]^{N} .
$$

Besides, we know that $\nabla \cdot\left(u_{\varepsilon}\right)=0$ in $\Omega_{\varepsilon}$, with $u_{\varepsilon} \cdot n=0$ on $\partial \Omega_{\varepsilon}$, and that $1_{\Omega_{\varepsilon}} u_{\varepsilon}$ converges strongly to $u$ in $\left[L^{2}(\Omega)\right]^{N}$. Passing to the limit yields

$$
\nabla \cdot u=0 \quad \text { in } \Omega
$$

Regrouping (3.19) and (3.20) we obtain the following homogenized system

$$
\left\{\begin{array}{l}
\text { Find }(u, p) \in\left[H_{0}^{l}(\Omega)\right]^{N} \times\left[L^{2}(\Omega) / \mathbb{R}\right] \quad \text { such that } \\
\nabla p-\mu \Delta u+\frac{\mu}{\sigma^{2}} M u=f \quad \text { in } \Omega \\
\nabla \cdot u=0 \quad \text { in } \Omega .
\end{array}\right.
$$

Because $M$ is a positive matrix, there exists a unique solution of (3.21). Then, all the subsequences of $\left(E_{c} u_{\varepsilon}, P_{c}\right)$ converge to the same limit. So the entire sequence converges. 
Remark 3.4. When the space dimension is $N=2$ or 3 , Theorem 3.4 can be easily generalized to the Navier-Stokes equations

$$
\left\{\begin{array}{l}
\text { Find }\left(u_{\varepsilon}, p_{\varepsilon}\right) \in H_{\varepsilon} \times\left[L^{2}\left(\Omega_{c}\right) / \mathbb{R}\right] \quad \text { such that } \\
\nabla p_{c}+u_{c} \cdot \nabla u_{c}-\mu \Delta u_{c}=f \quad \text { in } \Omega_{\varepsilon} \\
\nabla \cdot u_{c}=0 \quad \text { in } \Omega_{c} \\
u_{\varepsilon} \cdot n=0 \quad \text { on } \Gamma_{c} \\
\alpha_{c} u_{c}=2\left(\frac{\partial u_{c}}{\partial n} \cdot n\right) n-e\left(u_{c}\right) n \quad \text { on } \Gamma_{c} .
\end{array}\right.
$$

For $N=2$ or 3 , it is well known that there always exists a solution of system (3.22), which is unique at least for small values of $\|f\|_{L^{2}(\Omega)}$. For such a force $f$, the extension of the solution $\left(E_{\varepsilon} u_{\varepsilon}, P_{\varepsilon}\right)$ converges weakly in $\left[H_{0}^{1}(\Omega)\right]^{N} \times\left[L^{2}(\Omega) / \mathbb{R}\right]$ to $(u, p)$, which is the unique solution of the homogenized system (3.23)

$$
\left\{\begin{array}{l}
\text { Find } \left.(u, p) \in\left[H_{0}^{1}(\Omega)\right]^{N} \times\left[L^{2}(\Omega)\right] / \mathbb{R}\right] \quad \text { such that } \\
\nabla p+u \cdot \nabla u-\mu \Delta u+\frac{\mu}{\sigma^{2}} M u=f \quad \text { in } \Omega \\
\nabla \cdot u=0 \quad \text { in } \Omega .
\end{array}\right.
$$

More precisely, because the sequence $E_{\varepsilon} u_{\varepsilon}$ converges weakly to $u$ in $\left[H_{0}^{l}(\Omega)\right]^{N}$, the non-linear term $E_{\varepsilon} u_{\varepsilon} \cdot \nabla\left(E_{c} u_{\varepsilon}\right)$ converges strongly to $u \cdot \nabla u$ in $\left[H^{-1}(\Omega)\right]^{N}$, for $N=$ 2 or 3 . It is worth noticing that the local problem, the functions $\left(w_{k}^{c}, q_{k}^{e}\right)_{1 \leqq k \leqq N}$, introduced in Proposition 3.3, and therefore the matrix $M$, are exactly the same for Stokes and Navier-Stokes homogenization.

Remark 3.5. In [1] we gave several other theorems, including correctors for both the velocity and the pressure, and error estimates. We also generalized Theorem 3.4 to the case of a surface distribution of the holes. All those results still hold here, with slight changes in the proofs due to the difference between the Dirichlet boundary condition in [1] and the slip boundary condition under consideration in this paper. For brevity we do not repeat those proofs, and we refer to [1] for further details.

\section{Other Scalings}

In this section we consider scalings of the hole size and of the slip coefficient different from the critical one (3.1). First, we keep the size of the holes critical, and we examine the full range of values of the slip coefficient. Second, we briefly describe what happens for holes larger or smaller than critical, which is a mere reproduction of the situation described in Part II of [1]. 
PROPOSITION 4.1. Assume that the following scaling holds

$$
\left\{\begin{array}{c}
\lim _{\varepsilon \rightarrow 0} \alpha_{\varepsilon} a_{\varepsilon}=+\infty \\
\lim _{\varepsilon \rightarrow 0} \sigma_{\varepsilon}=\sigma
\end{array}\right.
$$

where $\sigma$ is a strictly positive constant. Then Theorem 3.4 is still true, and the matrix $M$ in the homogenized system (3.10) is obtained through a local problem with a Dirichlet boundary condition, which corresponds in Lemma 3.2 to the limit value $\alpha=+\infty$.

Proof: Because $\alpha_{c}$ is larger than $1 / a_{e}$, we can still derive from Lemma 2.4 the uniform coerciveness of the variational formulation $(2.10)$

$$
\left\|\nabla\left(E_{c} u_{\varepsilon}\right)\right\|_{L^{2}(\Omega)}^{2} \leqq C\left[\frac{1}{2} \int_{\Omega_{\varepsilon}}\left|e\left(u_{c}\right)\right|^{2}+\alpha_{e} \int_{\Gamma_{\epsilon}}\left|u_{c}\right|^{2}\right] .
$$

Thus, we obtain the same estimates, as in Proposition 3.1, for the velocity and the pressure.

On the other hand, we may define local problems as in Lemma 3.2, but with a Dirichlet boundary condition on $\partial T$. Then, in the same way we can construct test functions $\left(w_{k}^{k}, q_{k}^{k}\right)$ which satisfy the properties of Proposition 3.3, except that $w_{k}^{\varepsilon} \cdot n=0$ on $\Gamma_{\varepsilon}$ is replaced by $w_{k}^{c}=0$ on $\Gamma_{\varepsilon}$. In this case the matrix $M$ corresponds to an infinite value of $\alpha$. We repeat the proof of Theorem 3.4 with these new functions, and the only difference from Section 3 comes from the boundary contribution

$$
\int_{\Gamma_{c}} \phi u_{c} \cdot\left(\frac{\partial w_{k}^{\varepsilon}}{\partial n}+{ }^{\dagger} \nabla w_{k}^{\varepsilon} n\right)
$$

In order to pass to the limit in this new term (4.3) we point out that, because the boundary $\partial T$ is smooth, a standard regularity result implies that the solution $w_{k}$ of the local problem (3.6) or (3.8) satisfies

$$
\left(\frac{\partial w_{k}}{\partial n}+{ }^{t} \nabla w_{k} n\right) \in L^{2}(\partial T) .
$$

Rescaling (4.4) (see definitions (5.19) and (5.20) for the correct scaling of $w_{k}^{c}$ in terms of $w_{k}$ ) and summing over all the holes yields

$$
\left\|\left(\frac{\partial w_{k}^{c}}{\partial n}+{ }^{\top} \nabla w_{k}^{c} n\right)\right\|_{L^{2}\left(\Gamma_{\varepsilon}\right)}^{2} \leqq \frac{C}{a_{c}} .
$$


In the meantime from (4.2) we obtain

$$
\alpha_{\varepsilon} \int_{\Gamma_{\varepsilon}}\left|u_{\varepsilon}\right|^{2} \leqq C\|f\|_{L^{2}(\Omega)}^{2}
$$

Combining (4.5) and (4.6) we can bound (4.3)

$$
\left|\int_{\Gamma_{\varepsilon}} \phi u_{\varepsilon} \cdot\left(\frac{\partial w_{k}^{\varepsilon}}{\partial n}+{ }^{t} \nabla w_{k}^{\varepsilon} n\right)\right| \leqq \frac{C}{\left(a_{\varepsilon} \alpha_{\varepsilon}\right)^{1 / 2}}
$$

Thanks to the scaling (4.1) the upper bound in (4.7) tends to zero, so we are still able to pass to the limit and to obtain the homogenized system (3.10).

Proposition 4.2. Assume that the following scaling holds

$$
\left\{\begin{array}{c}
\lim _{\varepsilon \rightarrow 0} \alpha_{\varepsilon} a_{\varepsilon}=0 \\
\lim _{\varepsilon \rightarrow 0} \sigma_{\varepsilon}=\sigma
\end{array}\right.
$$

where $\sigma$ is a strictly positive constant. Moreover, we explicitly assume that we have a Dirichlet boundary condition on the large boundary $2 \Omega$ (but not on the boundaries of the holes $\Gamma_{\varepsilon}$ ). Then Theorem 3.4 is still true, and the matrix $M$ in the homogenized system (3.10) is obtained through a local problem with a mixed Dirichlet-Neumann boundary condition which corresponds in Lemma 3.2 to the limit value $\alpha=0$.

Proof: Because $\alpha_{\varepsilon}$ is smaller than $1 / a_{\varepsilon}$, we can no longer derive from Lemma 2.4 the uniform estimates for the velocity and the pressure. However, in the presence of a Dirichlet boundary condition on $\partial \Omega$, these estimates are still valid. Indeed, since $E_{\varepsilon} u_{\varepsilon}$ belongs to $\left[H_{0}^{\mathrm{l}}(\Omega)\right]^{N}$, a standard Korn inequality in $\Omega$ gives

$$
\left\|\nabla\left(E_{c} u_{\varepsilon}\right)\right\|_{L^{2}(\Omega)}^{2} \leqq C \int_{\Omega}\left|e\left(E_{\varepsilon} u_{\varepsilon}\right)\right|^{2}
$$

It is easy to see, using (4.9), that the conclusion of Proposition 3.1 still holds.

On the other hand, Lemma 3.2, which furnishes the form of the local problems, is still valid if the slip coefficient $\alpha$ is equal to zero. Then, as in Proposition 3.3, we construct test functions $\left(w_{k}^{\varepsilon}, q_{k}^{\varepsilon}\right)$ (note that the matrix $M$ is defined for $\alpha=0$ ). We repeat the proof of Theorem 3.4 with these new functions, and the only difference from Section 3 comes from the boundary contribution

$$
\alpha \int_{\Gamma_{\varepsilon}} \phi u_{\varepsilon} \cdot w_{k}^{e}
$$


If the spatial dimension is $N \geqq 3$, then using the trace Lemma 2.3 we bound (4.10)

$$
\begin{aligned}
\left|\alpha_{\varepsilon} \int_{\Gamma_{\varepsilon}} \phi u_{\varepsilon} \cdot w_{k}^{c}\right| & \leqq C \frac{\alpha_{\varepsilon} a_{\varepsilon}^{N-1}}{\varepsilon^{N}}\left\|w_{k}^{\varepsilon}\right\|_{H^{1}\left(\Omega_{c}\right)}\left\|u_{\varepsilon}\right\|_{H^{1}\left(\Omega_{\varepsilon}\right)} \\
& \leqq C \alpha_{\varepsilon} a_{\varepsilon} .
\end{aligned}
$$

Thanks to the scaling (4.8) the upper bound in (4.11) tends to zero, so we are still able to pass to the limit and to obtain the homogenized system (3.10). In the twodimensional case $N=2$, the definition of $\sigma_{\varepsilon}$ is slightly different (see (2.2)) and we cannot bound (4.10) by using the trace Lemma 2.3. Remark that Lemma 2.3 is not optimal here because it does not use the fact that both $u_{c}$ and $w_{k}^{k}$ have zero normal component on $\Gamma_{\epsilon}$. Nevertheless we can still prove that (4.10) tends to zero, and here is the trick. Note that, in $B_{1}-T$, the following trace inequality holds for any $w \in\left[H^{1}\left(B_{1}-T\right)\right]^{N}$, such that $w \cdot n=0$ on $\partial T$

$$
\|w\|_{L^{2}(\partial T)} \leqq C(T)\|\nabla w\|_{L^{2}\left(B_{1}-T\right)} .
$$

Then, rescaling (4.12), we obtain

$$
\left\|w_{k}^{\varepsilon}\right\|_{L^{2}\left(\partial T_{i}^{e}\right)}^{2} \leqq C a_{c}\left\|\nabla w_{k}^{\varepsilon}\right\|_{L^{2}\left(B_{i}^{a}-T_{i}^{e}\right)}^{2}
$$

and

$$
\left\|u_{\varepsilon}\right\|_{L^{2}\left(\partial T_{i}^{e}\right)}^{2} \leqq C a_{\varepsilon}\left\|\nabla u_{\varepsilon}\right\|_{L^{2}\left(B_{i}^{a}-T_{i}^{c}\right)}^{2}
$$

which implies

$$
\left\|w_{k}^{\varepsilon}\right\|_{L^{2}\left(\Gamma_{c}\right)}^{2} \leqq C a_{\varepsilon} \quad \text { and } \quad\left\|u_{c}\right\|_{L^{2}\left(\Gamma_{c}\right)}^{2} \leqq C a_{\varepsilon}\|f\|_{L^{2}(\Omega)}^{2}
$$

With these estimates, we obtain the same bound for $(4.10)$ as in the case $N \geqq 3$.

PROPOSITION 4.3. Assume that the size of the holes is smaller than the critical one, i.e., the ratio $\sigma_{\varepsilon}$, defined in (2.2), tends to infinity

$$
\lim _{\varepsilon \rightarrow 0} \sigma_{\varepsilon}=+\infty
$$

We emphasize that the scaling of the slip coefficient is free, but we explicitly assume that we have a Dirichlet boundary condition on the large boundary $\partial \Omega$ (not on $\Gamma_{\varepsilon}$ ). Let $\left(u_{\varepsilon}, p_{\varepsilon}\right)$ be the unique solution of the Stokes system (2.8). Then its extension 
$\left(E_{c} u_{\varepsilon}, P_{\varepsilon}\right)$ converges strongly in $\left[H_{0}^{1}(\Omega)\right]^{N} \times\left[L^{2}(\Omega) / \mathbb{R}\right]$ to $(u, p)$, which is the unique solution of the Stokes system

$$
\left\{\begin{array}{l}
\text { Find }(u, p) \in\left[H_{0}^{1}(\Omega)\right]^{N} \times\left[L^{2}(\Omega) / \mathbb{R}\right] \quad \text { such that } \\
\nabla p-\mu \Delta u=f \quad \text { in } \Omega \\
\nabla \cdot u=0 \quad \text { in } \Omega .
\end{array}\right.
$$

Proof: Because of the scaling (4.13), we can no longer derive uniformly bounded estimates of the solution with the help of Lemmas 2.4 and 2.5. Therefore, we need the Dirichlet boundary condition on $\partial \Omega$ in order to establish both Poincaré's and Korn's inequality with a uniform constant. For each $v \in\left[H_{0}^{1}(\Omega)\right]^{N}$, they give

$$
\|v\|_{L^{2}(\Omega)} \leqq C\|\nabla v\|_{L^{2}(\Omega)} \quad \text { and } \quad\|\nabla v\|_{L^{2}(\Omega)} \leqq C\|e(v)\|_{L^{2}(\Omega)} .
$$

Using (4.15) for $E_{c} u_{\varepsilon}$, it is easy to see that the conclusion of Proposition 3.1 still holds.

On the other hand, because of the small size of the holes (4.13), an easy but tedious computation (see [1]) shows that, for any positive value (including 0 and $+\infty$ ) of the slip coefficient $\alpha$, the convergence of the test functions $\left(w_{k}^{e}, q_{k}^{c}\right)$, defined in Proposition 3.3, is actually strong in $\left[H^{1}(\Omega)\right]^{N} \times L^{2}(\Omega)$. Thus, the function $\mu_{k}^{e}$, defined as $\left(\nabla q_{k}^{e}-\Delta w_{k}^{e}\right)$ in $\Omega_{\varepsilon}$ and zero elsewhere, converges strongly to zero in $\left[H^{-1}(\Omega)\right]^{N}$. This implies that no matrix $M$ appears when passing to the limit, and the homogenized system reduces to the Stokes equations (4.14). This fact can be checked straightforwardly (i.e., without the tedious computation of the test functions) as follows. In each cell $P_{i}^{e}$, let us define a function $\theta_{\varepsilon}$

$$
\begin{cases}\theta_{c}(r)=0 \text { for } 0 \leqq r \leqq a_{c}, & \theta_{c}(r)=\frac{r}{a_{c}}-1 \quad \text { for } \quad a_{c} \leqq r \leqq 2 a_{c}, \\ \theta_{c}(r)=1 \quad \text { elsewhere, for } \quad N \leqq 3 \\ \theta_{c}(r)=0 \text { for } 0 \leqq r \leqq a_{c}, & \theta_{\varepsilon}(r)=\frac{\log r-\log a_{\varepsilon}}{\log \varepsilon-\log a_{c}} \text { for } \quad a_{c} \leqq r \leqq \varepsilon, \\ \theta_{c}(r)=1 \quad \text { elsewhere, for } \quad N=2 .\end{cases}
$$

Then, for any $\phi \in D(\Omega)$, we have

$$
\theta_{\varepsilon} \phi \in H_{0}^{1}\left(\Omega_{\varepsilon}\right), \quad \text { and } \quad\left\|\theta_{\varepsilon} \phi-\phi\right\|_{H_{0}^{1}\left(\Omega_{\varepsilon}\right)} \leqq \frac{C}{\sigma_{\varepsilon}} .
$$

Using such test functions in the variational formulation (2.10), it is easy to see that the limit of (2.10) is a Stokes problem (without any additional term), because the sequence $\theta_{c} \phi$ converges strongly to $\phi$ in $H_{0}^{1}(\Omega)$. 
To prove that the solutions converge strongly, we observe that

$$
\frac{1}{2} \int_{\Omega_{c}}\left|e\left(u_{\varepsilon}\right)\right|^{2}+\alpha_{\varepsilon} \int_{\Gamma_{c}}\left|u_{\varepsilon}\right|^{2}=\int_{\Omega_{c}} f \cdot u_{\varepsilon} \rightarrow \int_{\Omega} f \cdot u=\int_{\Omega}|\nabla u|^{2} .
$$

Because $\nabla \cdot u=0$ in $\Omega$ and $u=0$ on $\partial \Omega$, an integration by parts shows that

$$
\frac{1}{2} \int_{\Omega}|e(u)|^{2}=\int_{\Omega}|\nabla u|^{2}
$$

Let $\chi_{\varepsilon}$ be the characteristic function of the set $\Omega_{\varepsilon}$ (i.e., $\chi_{\varepsilon}=1$ in $\Omega_{\varepsilon}$ and 0 elsewhere). We know that the sequence $e\left(E_{c} u_{\varepsilon}\right)$ converges weakly to $e(u)$ in $\left[L^{2}(\Omega)\right]^{N^{2}}$. Because $\chi_{\varepsilon}$ converges almost everywhere in $\Omega$ to 1 , the sequence $\chi_{\varepsilon} e\left(E_{\varepsilon} u_{c}\right)$ converges also weakly to $e(u)$ in $\left[L^{2}(\Omega)\right]^{N^{2}}$. Then, using the semilower continuity of the weak convergence, it follows from (4.16) and (4.17) that

$$
\lim _{\varepsilon \rightarrow 0} \int_{\Omega}\left|\chi_{\varepsilon} e\left(E_{\varepsilon} u_{\varepsilon}\right)\right|^{2}=\int_{\Omega}|e(u)|^{2} \quad \text { and } \quad \lim _{\varepsilon \rightarrow 0} \alpha_{\varepsilon} \int_{\Gamma_{\varepsilon}}\left|u_{\varepsilon}\right|^{2}=0 .
$$

The first limit in (4.18) implies the strong convergence of $\chi_{\varepsilon} e\left(E_{c} u_{\varepsilon}\right)$ to $e(u)$ in $\left[L^{2}(\Omega)\right]^{N^{2}}$. To obtain the same result for $e\left(E_{\varepsilon} u_{\varepsilon}\right)$ (without $\chi_{\varepsilon}$, we bound

$$
\left\|e(u)-e\left(E_{\varepsilon} u_{\varepsilon}\right)\right\|_{L^{2}(\Omega)} \leqq\left\|e(u)-e\left(E_{\varepsilon} u\right)\right\|_{L^{2}(\Omega)}+\left\|e\left(E_{\varepsilon} u\right)-e\left(E_{\varepsilon} u_{\varepsilon}\right)\right\|_{L^{2}(\Omega)} .
$$

Thanks to Lemma 2.2 on the extension operator $E_{\epsilon}$, we obtain

$$
\begin{aligned}
& \left\|e(u)-e\left(E_{\varepsilon} u_{\varepsilon}\right)\right\|_{L^{2}(\Omega)} \leqq\left\|e(u)-e\left(E_{\varepsilon} u\right)\right\|_{L^{2}(\Omega)}+C\left\|e(u)-e\left(u_{\varepsilon}\right)\right\|_{L^{2}\left(\Omega_{\varepsilon}\right)} \\
& \leqq\left\|e(u)-e\left(E_{\varepsilon} u\right)\right\|_{L^{2}(\Omega)}+C\left\|\chi_{\varepsilon} e(u)-\chi_{\varepsilon} e\left(E_{c} u_{\varepsilon}\right)\right\|_{L^{2}(\Omega)} \\
& \leqq\left\|e(u)-e\left(E_{\varepsilon} u\right)\right\|_{L^{2}(\Omega)}+C\left[\left\|e(u)-\chi_{\varepsilon} e(u)\right\|_{L^{2}(\Omega)}\right. \\
& \left.\quad+\left\|e(u)-\chi_{\varepsilon} e\left(E_{\varepsilon} u_{\varepsilon}\right)\right\|_{L^{2}(\Omega)}\right]
\end{aligned}
$$

The three terms in the right-hand side of (4.19) go to zero, as a consequence of Lemma 2.2 for the first one, the Lebesgue theorem of dominated convergence for the second one, and (4.18) for the last one. Hence we deduce the strong convergence of $e\left(E_{\varepsilon} u_{\varepsilon}\right)$ to $e(u)$ in $\left[L^{2}(\Omega)\right]^{N^{2}}$. Combined with the strong one in $\left[L^{2}(\Omega)\right]^{N}$, it yields the strong convergence of $E_{\varepsilon} u_{\varepsilon}$ to $u$ in $\left[H^{1}(\Omega)\right]^{N}$. Then, using Lemma 2.7 (which defines the extension of the pressure), it is easy to conclude that $P_{\varepsilon}$ converges strongly to $p$ in $L^{2}(\Omega) / \mathbb{R}$.

Proposition 4.4. Assume that the size of the holes is larger than the critical one, i.e., the ratio $\sigma_{\varepsilon}$, defined in (2.2), tends to zero

$$
\lim _{\varepsilon \rightarrow 0} \sigma_{\varepsilon}=0
$$


Let $\left(u_{\varepsilon}, p_{c}\right)$ be the unique solution of the Stokes system (2.8). After rescaling the velocity, the extension $\left(E_{\varepsilon} u_{\varepsilon} / \sigma_{\varepsilon}^{2}, P_{\varepsilon}\right)$ converges strongly in $\left[L^{2}(\Omega)\right]^{N} \times\left[L^{2}(\Omega) / \mathbb{R}\right]$ to $(u, p)$, which is the unique solution of Darcy's law

$$
\left\{\begin{array}{l}
\text { Find }(u, p) \in\left[L^{2}(\Omega)\right]^{N} \times\left[H^{1}(\Omega) / \mathbb{R}\right] \quad \text { such that } \\
u=\frac{M^{-1}}{\mu}(f-\nabla p) \quad \text { in } \Omega \\
\nabla \cdot u=0 \quad \text { in } \Omega \\
u \cdot n=0 \quad \text { on } \partial \Omega
\end{array}\right.
$$

where the matrix $M$ is still given by Definition 3.1, and depends on the slip coefficient $\alpha$ obtained through the following scaling

$$
\alpha=\lim _{\varepsilon \rightarrow 0} a_{\varepsilon} \alpha_{\varepsilon}
$$

If $\lim _{c \rightarrow 0}\left(a_{\varepsilon}^{N-1} \alpha_{\varepsilon}\right) / \varepsilon^{N}=0$, then the present proposition holds only if there is a Dirichlet boundary condition on $\partial \Omega$.

Proof: For the case of a Dirichlet boundary condition on $\Gamma_{\varepsilon}$, this proposition is proved in [1] (see Section 3.4). Using the ideas of the present paper, the proof for the slip boundary condition is a mere repetition of that in [1]. For the sake of brevity we do not repeat it here. However, let us emphasize that, even though the local problem is still given by Lemma 3.2 , the test functions $\left(w_{k}^{e}, q_{k}^{c}\right)$, involved in the proof, satisfy similar but weaker properties than that of Proposition 3.3. Furthermore, if $\lim _{c \rightarrow 0}\left(a_{c}^{N-1} \alpha_{\varepsilon}\right) / \varepsilon^{N}=0$, the Korn-type inequality of Lemma 2.4 fails to provide a uniform coercive estimate. In that case we need a Dirichlet boundary condition on $\partial \Omega$, in order to use the Korn inequality in $\left[H_{0}^{1}(\Omega)\right]^{N}$ (as in Proposition 4.3).

\section{Construction of the Test Functions}

This section is devoted to the proof of Lemma 3.2 and Proposition 3.3. We emphasize that, in this section, we consider only the critical scaling defined in (3.1). Actually, we focus on what is new compared to [1], where all the omitted details can be found. The treatment of the two-dimensional case is especially different from [1] in order clearly to show the source of the paradoxical result (3.9).

Proof of Lemma 3.2:

- $N \geqq 3$

For a fixed $\xi$ in $\mathbb{R}^{N}$, consider the Stokes system (5.1) 


$$
\left\{\begin{array}{l}
\nabla q_{\xi}-\Delta w_{\xi}=0 \quad \text { in } \mathbb{R}^{N}-T \\
\nabla \cdot w_{\xi}=0 \quad \text { in } \mathbb{R}^{N}-T \\
w_{\xi} \cdot n=0 \quad \text { on } \partial T \\
\alpha w_{\xi}=2\left(\frac{\partial w_{\xi}}{\partial n} \cdot n\right) n-e\left(w_{\xi}\right) n \quad \text { on } \partial T \\
w_{\xi}=\xi \quad \text { at } \infty .
\end{array}\right.
$$

In order to prove the existence and uniqueness of a solution we seek an equivalent minimization problem for $(5.1)$. Let $D^{1,2}\left(\mathbb{R}^{N}\right)$ be the completion, with respect to the $L^{2}$ norm of the gradient, of the space of all smooth functions with compact support

$$
D^{1,2}\left(\mathbb{R}^{N}\right)=\overline{D\left(\mathbb{R}^{N}\right)}\|\nabla \phi\|_{L_{2} \mathbb{R}^{N_{1}}} .
$$

If $N \geqq 3$, the following continuous embeddings hold (see, e.g., Ladyzhenskaya [16] for the first one, and Lemma 1.1, Chapter 4, in Lax-Phillips [17] for the second one):

$$
D^{1,2}\left(\mathbb{R}^{N}\right) \subset L^{2 N /(N-2)}\left(\mathbb{R}^{N}\right) \quad \text { and } \quad D^{1,2}\left(\mathbb{R}^{N}\right) \subset L_{\mathrm{loc}}^{2}\left(\mathbb{R}^{N}\right)
$$

Let $H_{\xi}=\left\{w \in\left[D^{1,2}\left(\mathbb{R}^{N}\right)\right]^{N} / \nabla \cdot w=0\right.$ in $\mathbb{R}^{N}$ and $(w+\xi) \cdot n=0$ on $\left.\partial T\right\}$, and, for positive $\alpha$, consider the following minimization problem

Find $w_{\xi}^{\prime}$ which achieves

$$
\inf _{w \in H_{\xi}}\left(\frac{1}{2} \int_{\mathbb{R}^{N}-T}|e(w)|^{2}+\alpha \int_{\partial T}|w+\xi|^{2}\right) .
$$

Because of (5.3), the functions in $H_{\xi}$ are equal to zero at infinity in the $L^{2 N /(N-2)}$ sense. Then, it is easy to check that (5.4) is equivalent to the Stokes problem (5.1): indeed, a coercive and strictly convex function on a closed, convex set admits a unique minimizer, and it is easy to see that $w_{\xi}=w_{\xi}^{\prime}+\xi$ is the unique solution of (5.1). In [1] we also proved that there exists a unique pressure $q_{\xi}$ solving (5.1) in $L^{2}\left(\mathbb{R}^{N}\right)$. Furthermore, an integration by parts shows that the drag force $F_{\xi}$ exerted on $T$ satisfies

$$
F_{\xi} \cdot \xi=\int_{\partial T}\left(q_{\xi} n-e\left(w_{\xi}\right) n\right) \cdot \xi=\frac{1}{2} \int_{\mathbb{R}^{N}-T}\left|e\left(w_{\xi}\right)\right|^{2}+\alpha \int_{\partial T}\left|w_{\xi}\right|^{2} .
$$


Decomposing $\xi$ in $\sum_{i=1}^{N} \xi_{i} e_{i}$, we obtain

$$
2^{N t} \xi M \xi=\sum_{1 \leqq i, j \leqq N} \xi_{i} \xi_{j} F_{i} \cdot e_{j}=\frac{1}{2} \int_{\mathbb{R}^{N}-T}\left|e\left(w_{\xi}\right)\right|^{2}+\alpha \int_{\partial T}\left|w_{\xi}\right|^{2} .
$$

Now, if we derive (5.5) with respect to $\alpha$, no contribution comes from $w_{\xi}$ because it achieves the minimum, and the derivative is positive, equal to $\int_{\partial T}\left|w_{\xi}\right|^{2}$. This gives the proof that $\alpha_{1} \leqq \alpha_{2}$ implies $M\left(\alpha_{1}\right) \leqq M\left(\alpha_{2}\right)$. Going back to (5.4) it is easy to see that the limit case when $\alpha$ tends to infinity corresponds to a Dirichlet boundary condition on $\partial T$. As in [1], using the fundamental solution of the Stokes operator in $\mathbb{R}^{N}$ (see [12] and [16]), we obtain asymptotic expansions at infinity of the solution of $(5.1)$

$$
\left\{\begin{array}{l}
w_{\xi}=\xi-\frac{1}{2 S_{N} r^{N-2}}\left[\frac{F_{\xi}}{N-2}+\left(F_{\xi} \cdot e_{r}\right) e_{r}\right]+O\left(\frac{1}{r^{N-1}}\right) \\
q_{\xi}=-\frac{1}{S_{N} r^{N-1}}\left(F_{\xi} \cdot e_{r}\right)+O\left(\frac{1}{r^{N}}\right) \\
\nabla w_{\xi}=O\left(\frac{1}{r^{N-1}}\right) \\
\frac{\partial w_{\xi}}{\partial r}-q_{\xi} e_{r}=\frac{1}{2 S_{N} r^{N-1}}\left[F_{\xi}+N\left(F_{\xi} \cdot e_{r}\right) e_{r}\right]+O\left(\frac{1}{r^{N}}\right) .
\end{array}\right.
$$

These expansions are accurate if the drag force $F_{\xi}$ is non-zero. That is always the case: by comparison with the small ball $B_{r_{0}}$ included in $T$, it is not difficult to show that $F_{\xi} \cdot \xi>0$ for any non-zero $\xi$.

- $N=2$

This case is completely different from the previous one, because there is no solution of (5.1) in two dimensions (this is the well-known Stokes paradox). That is why we seek a solution of the Stokes equations with a logarithmic growth at infinity:

$$
\left\{\begin{array}{l}
\nabla q_{\xi}-\Delta w_{\xi}=0 \quad \text { in } \mathbb{R}^{2}-T \\
\nabla \cdot w_{\xi}=0 \quad \text { in } \mathbb{R}^{2}-T \\
w_{\xi} \cdot n=0 \quad \text { on } \partial T \\
\alpha w_{\xi}=2\left(\frac{\partial w_{\xi}}{\partial n} \cdot n\right) n-e\left(w_{\xi}\right) n \quad \text { on } \partial T \\
w_{\xi}=(\log r) \xi \quad \text { at } \infty .
\end{array}\right.
$$

If $T$ is a ball $B_{a}$ of radius $a$, we can compute an exact solution of (5.7), denoted by $\left(q_{\xi}^{0}, w_{\xi}^{0}\right)$ 


$$
\left\{\begin{array}{l}
w_{\xi}^{0}=\left(\xi \cdot e_{r}\right) r^{2} f(r) e_{r}+g(r) \xi \\
q_{\xi}^{0}=\left(\xi \cdot e_{r}\right) r h(r)
\end{array}\right.
$$

$$
\begin{aligned}
& \text { with }\left\{\begin{array}{l}
f(r)=\frac{1}{r^{2}}\left(-1+\frac{A}{r^{2}}\right) \\
g(r)=\log r-\frac{A}{2 r^{2}}+B \\
h(r)=-\frac{2}{r^{2}}
\end{array}\right. \\
& \text { with }\left\{\begin{array}{l}
A=\frac{a^{2}}{1+\frac{2}{\alpha a}} \\
B=\frac{4+\alpha a}{4+2 \alpha a}-\log a
\end{array}\right.
\end{aligned}
$$

Moreover, the drag force $F_{\xi}^{0}$ is equal to $4 \pi \xi$, for any value of $\alpha$ and $a$. In order to extend that result to an arbitrary shape of the obstacle $T$, we seek a solution of (5.7) in the form $w_{\xi}=w_{\xi}^{0}+w_{\xi}^{\prime}$ and $q_{\xi}=q_{\xi}^{0}+q_{\xi}^{\prime}$ where, from now on, $\left(q_{\xi}^{0}, w_{\xi}^{0}\right)$ denotes the Stokes solution for the ball $B_{1}$ with a Dirichlet boundary condition (i.e., $\alpha=+\infty$ in formulae (5.10)). Let $\delta_{B_{1}}$ be the homogeneous, unit mass, surface measure concentrated on $\partial B_{1}$. An easy computation shows that $q_{\xi}^{0} e_{r}-\left(\partial w_{\xi}^{0} / \partial r\right)$ is equal to $-2 \xi$ on the circle $\partial B_{1}$. By subtraction $\left(q_{\xi}^{\prime}, w_{\xi}^{\prime}\right)$ is a solution of the following system

$$
\left\{\begin{array}{l}
\nabla q_{\xi}^{\prime}-\Delta w_{\xi}^{\prime}=-\left(q_{\xi}^{0} e_{r}-\frac{\partial w_{\xi}^{0}}{\partial r}\right) \delta_{B_{1}}=2 \xi \delta_{B_{1}} \quad \text { in } \mathbb{R}^{2}-T \\
\nabla \cdot w_{\xi}^{\prime}=0 \quad \text { in } \mathbb{R}^{2}-T \\
w_{\xi}^{\prime} \cdot n=0 \quad \text { on } \partial T \\
\alpha w_{\xi}^{\prime}=2\left(\frac{\partial w_{\xi}^{\prime}}{\partial n} \cdot n\right) n-e\left(w_{\xi}^{\prime}\right) n \quad \text { on } \partial T \\
w_{\xi}=o(\log r) \quad \text { at } \quad \infty \quad\left(\text { i.e., } \frac{\left|w_{\xi}\right|}{\log r} \rightarrow 0\right) .
\end{array}\right.
$$

We shall give a minimization formulation to system (5.11) as we did for (5.1). Let $D^{1,2}\left(\mathbb{R}^{2}\right)$ be the completion, with respect to the $L^{2}$ norm of the gradient, of the space of all smooth functions with compact support in $\mathbb{R}^{2}$. Unfortunately, there is no Sobolev embedding for $D^{1,2}\left(\mathbb{R}^{2}\right)$ ( an important consequence of that fact is that functions of $D^{1,2}\left(\mathbb{R}^{2}\right)$ do not go to zero at infinity; for example, constants belong 
to $D^{1,2}\left(\mathbb{R}^{2}\right)$ ). Nevertheless, O. A. Ladyzhenskaya in [16] proved that any smooth function $\phi$ with compact support in $\mathbb{R}^{2}-B_{1}$ satisfies

$$
\left\|\frac{\phi}{r \log r}\right\|_{L^{2}\left(\mathbb{R}^{2}-B_{1}\right)} \leqq 4\|\nabla \phi\|_{L^{2}\left(\mathbf{R}^{2}-B_{1}\right)} .
$$

Thus, we can deduce a continuous embedding, weaker than (5.3)

$$
D^{1,2}\left(\mathbb{R}^{2}\right) \subset\left\{\phi \in L_{\mathrm{loc}}^{2}\left(\mathbb{R}^{2}\right) / \frac{\phi}{(r+1) \log (r+2)} \in L^{2}\left(\mathbb{R}^{2}\right)\right\} .
$$

Functions of $D^{1,2}\left(\mathbb{R}^{2}\right)$ behave like $o(\log r)$ at infinity, in the sense of $(5.12)$, because $\log r$ does not belong to the space on the right-hand side of (5.12). Let $H_{\xi}=\{w \in$ $\left[D^{1,2}\left(\mathbb{R}^{2}\right)\right]^{2} / \nabla \cdot w=0$ in $\mathbb{R}^{2}$ and $w \cdot n=0$ on $\left.\partial T\right\}$.

Consider now the following minimization problem

$$
\text { Find } w_{\xi}^{\prime} \text { which achieves }
$$

$$
\inf _{w \in H_{\xi}}\left(\frac{1}{4} \int_{\mathbb{R}^{2}-T}|e(w)|^{2}+\frac{\alpha}{2} \int_{\partial T}|w|^{2}-2 \int_{\partial B_{1}} w \cdot \xi\right) .
$$

It is easy to check that (5.13) is equivalent to the Stokes system (5.11). As (5.13) admits a unique minimizer $w_{\xi}^{\prime}$, we conclude that $w_{\xi}=w_{\xi}^{\prime}+w_{\xi}^{0}$ is the unique solution of (5.7). Now it remains to show that the drag force $F_{\xi}$ is always equal to $4 \pi \xi$ (this paradoxical result has already been pointed out by R. Finn and D. Smith in [13]). We have

$$
F_{\xi}=\int_{\partial T}\left(q_{\xi} n-\frac{\partial w_{\xi}}{\partial n}\right)=\int_{\partial B_{R}}\left(\frac{\partial w_{\xi}}{\partial r}-q_{\xi} e_{r}\right) \quad \text { for any } \quad R \geqq 1 .
$$

Decomposing $w_{\xi}$ as $w_{\xi}^{0}+w_{\xi}^{\prime}$ we obtain

$$
F_{\xi}=4 \pi \xi+\int_{\partial B_{R}}\left(\frac{\partial w_{\xi}^{\prime}}{\partial r}-q_{\xi}^{\prime} e_{r}\right) \quad \text { for any } \quad R \geqq 1 .
$$

Let $w_{\xi^{\prime}}^{0}$ be the same function as $w_{\xi}^{0}$, but for $\xi^{\prime} \neq \xi$. From $(5.8)-(5.10)$ we know that $w_{\xi^{\prime}}^{0}$ is equal to 0 on $\partial B_{1}$ and to $\log r[1+o(1)] \xi^{\prime}$ at infinity. Multiplying equation $(5.11)$ by $w_{\xi^{\prime}}^{0} / \log R$ and integrating by parts over $B_{R}-B_{1}$ yields

$$
\frac{1}{\log R} \int_{B_{R}-B_{1}} \nabla w_{\xi^{\prime}}^{0} \cdot \nabla w_{\xi}^{\prime}=\left[\int_{\partial B_{R}}\left(\frac{\partial w_{\xi}^{\prime}}{\partial n}-q_{\xi}^{\prime} n\right) \cdot \xi^{\prime}\right][1+o(1)] .
$$


For any $\xi^{\prime}$ we can bound $(5.15)$

$$
\left|\int_{\partial B_{R}}\left(\frac{\partial w_{\xi}^{\prime}}{\partial n}-q_{\xi}^{\prime} n\right) \cdot \xi^{\prime}\right| \leqq \frac{1}{\log R}\left\|\nabla w_{\xi^{\prime}}^{0}\right\|_{L^{2}\left(B_{R}-B_{1}\right)}\left\|\nabla w_{\xi}^{\prime}\right\|_{L^{2}\left(\mathbb{R}^{2}-T\right)}
$$

$$
\leqq \frac{C}{(\log R)^{1 / 2}}
$$

When $R$ tends to infinity, we deduce from (5.14) and (5.16)

$$
F_{\xi}=4 \pi \xi
$$

We now turn to the construction of the test functions, which, roughly speaking, are obtained by rescaling the solutions of the local problems, and expanding them by $P_{i}^{\varepsilon}$-periodicity in the whole domain $\Omega$.

Proof of Proposition 3.3: We decompose each cell $P_{i}^{\varepsilon}$ in smaller subdomains. We set

$$
\bar{P}_{i}^{\varepsilon}=T_{i}^{\varepsilon} \cup \bar{C}_{i}^{\prime} \cup \bar{D}_{i}^{\varepsilon} \cup \bar{K}_{i}^{\varepsilon}
$$

where $C_{i}^{c}$ is the open ball of radius $\varepsilon / 2$ centered in $P_{i}^{c}$, and perforated by $T_{i}^{c}, D_{i}^{\varepsilon}$ is equal to $B_{i}^{\varepsilon}$ perforated by $\bar{C}_{i}^{\prime \varepsilon} \cup T_{i}^{\varepsilon}$, and $K_{i}^{\varepsilon}$ is the remainder, i.e., the corners of $P_{i}^{e}$.

Let $\left(w_{k}, q_{k}\right)_{1 \leqq k \leqq N}$ be the solutions of the local problem defined in Lemma 3.2. We define the functions $\left(w_{k}^{\varepsilon}, q_{k}^{\varepsilon}\right)_{1 \leqq k \leq N}$ by

- For each cube $P_{i}^{\varepsilon}$ which meets $\partial \Omega$

$$
\left\{\begin{array}{l}
w_{k}^{c}=e_{k} \\
q_{k}^{c}=0
\end{array} \quad \text { in } P_{i}^{\varepsilon} \cap \Omega\right.
$$

- For each cube $P_{i}^{c}$ entirely included in $\Omega$

$$
\begin{aligned}
& \left\{\begin{array}{l}
w_{k}^{e}=e_{k} \\
q_{k}^{\varepsilon}=0
\end{array} \quad \text { in } K_{i}^{c}\right. \\
& \left\{\begin{array}{l}
\nabla q_{k}^{e}-\Delta w_{k}^{e}=0 \\
\nabla \cdot w_{k}^{e}=0
\end{array} \text { in } D_{i}^{e}\right.
\end{aligned}
$$




$$
\begin{aligned}
& \left\{\begin{array}{l}
w_{k}^{c}(x)=w_{k}\left(\frac{x}{a_{\varepsilon}}\right) \\
q_{k}^{\varepsilon}(x)=\frac{1}{a_{\varepsilon}} q_{k}\left(\frac{x}{a_{\varepsilon}}\right) \text { in } C_{i}^{\prime \varepsilon} \text { for } N \geqq 3
\end{array}\right. \\
& \left\{\begin{array}{l}
w_{k}^{c}(x)=\frac{1}{\log \frac{\varepsilon}{2 a_{\varepsilon}} w_{k}\left(\frac{x}{a_{\varepsilon}}\right)} \\
q_{k}^{e}(x)=\frac{1}{a_{\varepsilon} \log \left(\frac{\varepsilon}{2 a_{\varepsilon}}\right)} q_{k}\left(\frac{x}{a_{\varepsilon}}\right) \\
\left\{\begin{array}{l}
\nabla q_{k}^{\varepsilon}-\Delta w_{k}^{c}=0 \\
\nabla \cdot w_{k}^{c}=0
\end{array} \quad \text { in } C_{i}^{c}\right.
\end{array}\right.
\end{aligned}
$$

where $\left(w_{k}^{\varepsilon}, q_{k}^{\varepsilon}\right) \in\left[H^{1}\left(P_{i}^{c}\right)\right]^{N} \times L^{2}\left(P_{i}^{c}\right)$.

Using the properties of the local solutions (including the asymptotic expansions in the case $N \geqq 3$, and the decomposition in a "spherical" part and a small remainder in the case $N=2$ ), we can easily prove, as we did in [1], that

(i) $q_{k}^{\varepsilon} \rightarrow 0$ in $L^{2}(\Omega) / \mathbb{R}$ weakly.

(ii) $w_{k}^{\varepsilon} \rightarrow e_{k}$ in $\left[H^{1}(\Omega)\right]^{N}$ weakly, and $\nabla \cdot w_{k}^{\varepsilon}=0$ in $\Omega$.

(iii) $w_{k}^{c} \cdot n=0$ and $\left(\alpha / a_{c}\right) w_{k}^{c}=2\left[\left(\partial w_{k}^{c} / \partial n\right) \cdot n\right] n-e\left(w_{k}^{c}\right) n$ on $\Gamma_{\varepsilon}$.

Basically the main fact is to remark that

$$
\left\{\begin{array}{l}
\left\|\nabla w_{k}\right\|_{L^{2}\left(\mathbb{R}^{N}\right)}<\infty \quad \text { for } \quad N \geqq 3 \\
\left\|\nabla w_{k}\right\|_{L^{2}\left(B_{R}\right)}^{2}=2 \pi \log R[1+o(1)] \quad \text { for } \quad N=2 .
\end{array}\right.
$$

Then by rescaling (5.20), and summing over cells $P_{i}^{c}$ (the number of which is of order $\varepsilon^{-N}$ ), we obtain

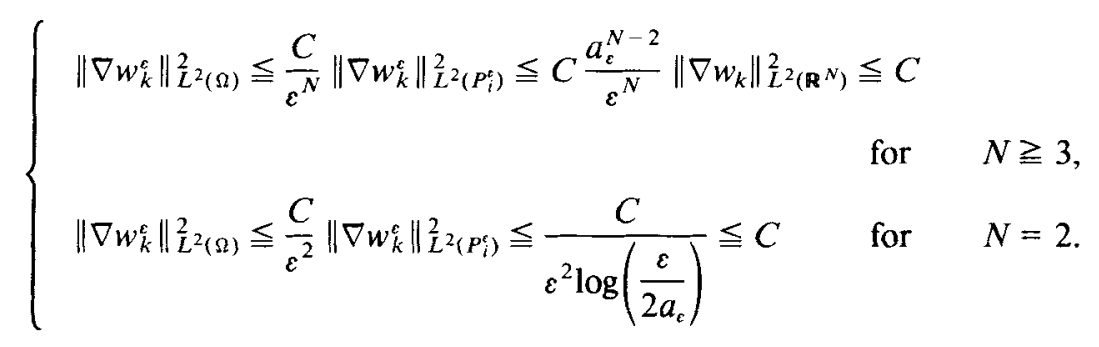

For the sake of simplicity we omitted in (5.21) the estimates of $\nabla w_{k}^{\varepsilon}$ in $D_{i}^{\varepsilon}$ and in $T_{i}^{\varepsilon}$, which anyway turn out to be similar (and even stronger) to those in $C_{i}^{\prime c}$ (see [1] for details). 
The main point to prove in Proposition 3.3 is the last statement (iv), namely (iv) Let $\mu_{k}^{\varepsilon}=\nabla q_{k}^{\varepsilon}-\Delta w_{k}^{\varepsilon} \in\left[H^{-1}\left(\Omega_{\varepsilon}\right)\right]^{N}$. Then $\mu_{k}^{c}$ has compact support in $\Omega_{\varepsilon}$. Moreover, extending $\mu_{k}^{\varepsilon}$ by 0 in $\Omega-\Omega_{\varepsilon}$ we have

$$
\mu_{k}^{\varepsilon} \rightarrow \frac{1}{\sigma^{2}} M e_{k} \quad \text { in } \quad\left[H^{-1}(\Omega)\right]^{N} \quad \text { strongly }
$$

First, thanks to the definitions (5.18) and (5.19), the function $\left(\nabla q_{k}^{e}-\Delta w_{k}^{e}\right)$ is equal to zero everywhere in $\Omega_{\varepsilon}$ except on the boundaries of $C_{i}^{\prime c}$ and $D_{i}^{c}$. Thus $\mu_{k}^{e}$ has compact support in $\Omega_{c}$ as claimed in (iv). Second, we shall prove that $\mu_{k}^{c}$ converges strongly to $\left(1 / \sigma^{2} 2^{N}\right) F_{k}$ in $\left[H^{-1}(\Omega)\right]^{N}$. However we only sketch this proof, because it is a mere repetition of Lemmas 2.3 .3 and 2.3.7 in [1]. A tedious computation yields the strong $L^{2}$ convergence of $\left(q_{k}^{c}, \nabla w_{k}^{c}\right)$ to zero in the union of the $D_{i}^{\varepsilon}$. Thus the part of $\nabla q_{k}^{\varepsilon}-\Delta w_{k}^{\varepsilon}$ concentrated on the boundaries of the $D_{i}^{\varepsilon}$ converges strongly to zero in $\left[H^{-1}(\Omega)\right]^{N}$. On the other hand, because of the definition of the function $\mu_{k}^{\varepsilon}$ we may skip the contribution of $\nabla q_{k}^{\varepsilon}-\Delta w_{k}^{\varepsilon}$ on the boundaries $\partial C_{i}^{\prime \varepsilon} \cap$ $\partial T_{i}^{c}$. It only remains to prove that

$$
\sum_{i=1}^{N(c)}\left(\frac{\partial w_{k}^{e}}{\partial r_{i}}-q_{k}^{\varepsilon} e_{r}^{i}\right) \delta_{i}^{c / 2}
$$

(where $\delta_{i}^{\varepsilon / 2}$ is the unit mass concentrated on the sphere $\partial C_{i}^{\prime \varepsilon} \cap \partial D_{i}^{\varepsilon}$ ) converges strongly to $\left(1 / \sigma^{2} 2^{N}\right) F_{k}$ in $\left[H^{-1}(\Omega)\right]^{N}$. Introducing the solutions of the local problem leads to

$$
\begin{aligned}
\sum_{i=1}^{N(\varepsilon)}\left(\frac{\partial w_{k}^{c}}{\partial r_{i}}-q_{k}^{\varepsilon} e_{r}^{i}\right) \delta_{i}^{c / 2} & \\
(5.22)= & \left\{\begin{array}{lll}
\sum_{i=1}^{N(\varepsilon)} \frac{2^{N}}{4 \sigma^{2} S_{N}}\left[F_{k}+N\left(F_{k} \cdot e_{r}^{i}\right) e_{r}^{i}\right] \varepsilon \delta_{i}^{\varepsilon / 2}[1+o(1)] & \text { for } & N \geqq 3 \\
\sum_{i=1}^{N(\varepsilon)} \frac{2}{\sigma^{2}} e_{k} \varepsilon \delta_{i}^{\varepsilon / 2}[1+o(1)] & \text { for } & N=2 .
\end{array}\right.
\end{aligned}
$$

Then using a lemma of D. Cioranescu and F. Murat (see [9]), which asserts that

$$
\left\{\begin{array}{l}
\sum_{i=1}^{N(\varepsilon)} \varepsilon \delta_{i}^{\varepsilon} \rightarrow \frac{S_{N}}{2^{N}} \quad \text { in } H^{-1}(\Omega) \text { strongly } \\
\sum_{i=1}^{N(\varepsilon)} \varepsilon \delta_{i}^{\varepsilon}\left(e_{k} \cdot e_{r}^{i}\right) e_{r}^{i} \rightarrow \frac{S_{N}}{N 2^{N}} e_{k} \quad \text { in }\left[H^{-1}(\Omega)\right]^{N} \text { strongly, }
\end{array}\right.
$$


we prove the desired convergence ( see [1] for details)

$$
\sum_{i=1}^{N(c)}\left(\frac{\partial w_{k}^{\varepsilon}}{\partial r_{i}}-q_{k}^{\varepsilon} e_{r}^{i}\right) \delta_{i}^{c / 2} \rightarrow \frac{1}{\sigma^{2} 2^{N}} F_{k} .
$$

Acknowledgments. Part of this research was supported by DARPA Grant No. F49620-87-C-0065. The author wishes to thank the Courant Institute of Mathematical Sciences for its hospitality. The author is also indebted to R. V. Kohn, F. Murat, and J. P. Puel for their unfailing help throughout this work.

\section{Bibliography}

[1] Allaire, G., Homogenization of the Navier-Stokes equations in open sets perforated with tiny holes, Arch. Rat. Mech. Anal. 113, 1991, Part I, pp. 209-259, and Part II, pp. 261-298.

[2] Allaire, G., Homogenization of the Stokes flow in a connected porous medium, Asymptotic Analysis 2, 1989, pp. 203-222.

[3] Allaire, G., Homogenization of the Navier-Stokes equations and derivation of Brinkman's law, Segundo Congreso Franco-Chileno y Latinoamericano de Matematicas Aplicadas, December 4$8,1989$.

[4] Allaire, G., Continuity of the Darcy's law in the low-volume fraction limit, to appear.

[5] Bensoussan, A., Lions, J. L., and Papanicolaou, G., Asymptotic Analysis for Periodic Structures, North-Holland, 1978.

[6] Brillard, A., Asymptotic analysis of incompressible and viscous fluid flow through porous media. Brinkman's law via epi-convergence methods, Ann. Fac. Sci. Toulouse 8, 1986, pp. 225-252.

[7] Brillard, A., Asymptotic analysis of two elliptic equations with oscillating terms, Math. Model. and Num. Anal. 22, 1988, pp. 187-216.

[8] Brinkman, H. C., A calculation of the viscous force exerted by a flowing fluid on a dense swarm of particles, Appl. Sci. Res. A1, 1947, pp. 27-34.

[9] Cioranescu, D., and Murat, F., Un terme étrange venu d'ailleurs, in Nonlinear Partial Differential Equations and Their Applications, Collège de France Seminar, Vols. 2 and 3, H. Brézis and J. L. Lions, eds., Research Notes in Mathematics 60 and 70, Pitman, London, 1982.

[10] Cioranescu, D., and Saint Jean Paulin, J., Homogenization in open sets with holes, Journal of Mathematical Analysis and Applications, 71, 1979, pp. 590-607.

[11] Conca, C., On the application of the homogenization theory to a class of problems arising in fluid mechanics, J. Math. Pures et Appl. 64, 1985, pp. 31-75.

[12] Finn, R., Mathematical questions relating to viscous fluid flow in an exterior domain, Rocky Mountain J. Math. 3, 1973, pp. 107-140.

[13] Finn, R., and Smith, D., On the linearized hydrodynamical equations in two dimensions, Arch. Rat. Mech. Anal. 25, 1967, pp. 1-25.

[14] Girault, V., and Raviart, P. A., Finite Elements Methods For Navier-Stokes Equations, Springer Series in Computational Mathematics 5, Springer-Verlag, Berlin, 1986.

[15] Keller, J. B., Darcy's law for flow in porous media and the two-space method, Nonlinear P.D.E.s in Engineering and Applied Science, Lecture Notes in Pure and Appl. Math. 54, R. Sternberg et al., eds., pp. 429-443, Dekker, New York, 1980.

[16] Ladyzhenskaya, O. A., The Mathematical Theory of Viscous Incompressible Flow, Gordon and Breach, New York, 1969.

[17] Lax, P. D., and Phillips, R. S., Scattering theory, in Pure and Applied Mathematics 26, Academic Press, New York, 1967.

[18] Levy, T., Fluid flow through an array of fixed particles, Int. J. Engin. Sci. 21, 1983, pp. 11-23.

[19] Lions, J. L., Some methods in the mathematical analysis of systems and their control, Beijing, Gordon and Breach, New York, 1981. 
[20] Lipton, R., and Avellaneda, M., A Darcy law for slow viscous flow past a stationary array of bubbles, Proc. Roy. Soc., Edinburgh, 114A, 1990, pp. 71-79.

[21] Marcenko, V. A., and Hrouslov, E. Ja., Boundary problems in domains with finely granulated boundaries (in Russian), Naukova Dumka, Kiev, 1974.

[22] Murat, F., H-convergence, Séminaire d'Analyse Fonctionnelle et Numérique de l'Université d'Alger, mimeographed notes, 1978.

[23] Rubinstein, J., On the macroscopic description of slow viscous flow past a random array of spheres, J. Stat. Phys. 44, 1986, pp. 849-863.

[24] Sanchez-Palencia, E., On the asymptotics of the fluid flow past an array of fixed obstacles, Int. J. Engin. Sci. 20, 1982, pp. 1291-1301.

[25] Sanchez-Palencia, E., Non-homogeneous media and vibration theory, Lecture Notes in Physics 127, Springer-Verlag, Berlin, 1980.

[26] Tartar, L., Convergence of the homogenization process, Appendix in [25].

[27] Tartar, L., Cours Peccot au Collegge de France, unpublished, March 1977.

Received June 1990. 